\title{
Influence of platelet-rich plasma (PRP) analogues on healing and clinical outcomes following anterior cruciate ligament (ACL) reconstructive surgery: a systematic review
}

\author{
Jonathon McRobb ${ }^{1}$ - Khawaja Hasan Kamil' $\cdot$ Imran Ahmed ${ }^{2} \cdot$ Fatema Dhaif $^{2} \cdot$ Andrew Metcalfe $^{2}$
}

Received: 23 August 2021 / Accepted: 29 December 2021 / Published online: 12 January 2022

(c) The Author(s) 2022

\begin{abstract}
Purpose To systematically review the effect of PRP on healing (vascularization, inflammation and ligamentization) and clinical outcomes (pain, knee function and stability) in patients undergoing ACL reconstruction and compare the preparation and application of PRP.

Methods Independent systematic searches of online databases (Medline, Embase and Web of Science) were conducted following PRISMA guidelines (final search 10th July 2021). Studies were screened against inclusion criteria and risk of bias assessed using Critical appraisal skills programme (CASP) Randomised controlled trial (RCT) checklist. Independent data extraction preceded narrative analysis.

Results 13 RCTs were included. The methods of PRP collection and application were varied. Significant early increases in rate of ligamentization and vascularisation were observed alongside early decreases in inflammation. No significant results were achieved in the later stages of the healing process. Significantly improved pain and knee function was found but no consensus reached.

Conclusions PRP influences healing through early vascularisation, culminating in higher rates of ligamentization. Longterm effects were not demonstrated suggesting the influence of PRP is limited. No consensus was reached on the impact of PRP on pain, knee stability and resultant knee function, providing avenues for further research. Subsequent investigations could incorporate multiple doses over time, more frequent observation and comparisons of different forms of PRP. The lack of standardisation of PRP collection and application techniques makes comparison difficult. Due to considerable heterogeneity, $\left(I^{2}>50 \%\right)$, a formal meta-analysis was not possible highlighting the need for further high quality RCTs to assess the effectiveness of PRP. The biasing towards young males highlights the need for a more diverse range of participants to make the study more applicable to the general population.
\end{abstract}

Trail registration CRD42021242078CRD, 15th March 2021, retrospectively registered.

Keywords Platelet-rich-plasma $\cdot$ Anterior cruciate ligament reconstruction $\cdot$ Biologic augmentation $\cdot$ Systematic review

Jonathon McRobb

Jonathon.mcrobb@warwick.ac.uk

Khawaja Hasan Kamil

Hasan.kamil@warwick.ac.uk

Imran Ahmed

Imran.ahmed4@nhs.net

Fatema Dhaif

fdhaif13@gmail.com

Andrew Metcalfe

a.metcalfe@warwick.ac.uk

1 Warwick Medical School, Medical School Building, Coventry CV4 7HL, UK

2 Warwick Clinical Trials Unit, Coventry CV4 7AL, UK

\section{Introduction}

Anterior cruciate ligament (ACL) rupture is a common injury; 68.6 per 100,000 person years [1]. It often requires surgical intervention and rigorous physiotherapy to allow patients to return to their normal activities. Particularly prevalent in sport, excessive knee rotation can cause ACL rupture, leading to knee pain and instability [2]. The avascular nature of ligamentous tissue and stabilising muscle atrophy result in long recovery times [3]. This inhibits Activities of daily living (ADLs) and can result in prolonged periods of absence from work or /sport. 
Platelet-rich plasma (PRP) is a biological augmentation reported to aid healing in damaged cartilage, tendon and ligamentous structures [4]. Resulting from blood separation, PRP consists of autologous mixtures of concentrated platelets and growth factors. Associated with recruitment and proliferation of cells and stimulating angiogenesis [5], early studies have shown improvements in healing and subsequent recovery, indicating use for injuries that require longer recovery periods [6]. Further to this PRP has been reported to reduce pain during recovery in orthopaedic setting [7]. Pain is a major barrier in returning to previous levels of function, either due to effect on mental well-being or ability to complete rehabilitation exercises [8]. In an injury that requires substantial physiotherapy, PRP could improve recovery by facilitating better adherence to physiotherapy protocols, improving knee stability and function.

Much of the literature investigates PRP use in the context of oral and orthopaedic procedures; however, the literature has failed to reach a consensus on its efficacy in ACL reconstruction [9]. This study aims to systematically review the current literature to determine the influence of PRP on the healing and clinical outcomes of ACL reconstructive surgery and compare the methods of PRP collection and application.

\section{Methodology}

The review was conducted in accordance with the Preferred reporting items for systematic review and meta-analysis (PRISMA) guidelines. The systematic review was registered on PROSPERO; registration number CRD42021242078. The protocol was designed after consultation with a medical librarian. Studies were then selected based on eligibility criteria (Fig. 1).

\section{Inclusion}

- Randomised controlled trial reporting the effect of PRP on ACL reconstructive surgery outcomes

- Published in English between 2005 and 2021

- Evaluated knee pain, stability, function, vascularisation, inflammation or ligamentization

- Study conducted on humans of any age or gender

\section{Exclusion}

- Reviews and conference abstracts

- Not discussing ACL reconstruction or PRP

\section{Search strategy}

A systematic search of Medline, Embase and Web of Science [Figs. 2, 3, 4 respectively] using key words was conducted on 10th of July 2021. Results were exported to Endnote and independently de-duplicated by both authors. Study title and abstracts were then screened against eligibility criteria independently by both authors. Discordance led to inclusion for full text screening to ensure no study was prematurely excluded. Full text screening of the remaining studies was conducted independently by both authors and disagreements remedied by discussion.

\section{Quality assessment}

All included studies were critically appraised by both authors independently using the Critical appraisal skills programme (CASP) RCT checklist [10] with disagreements remedied by discussion. Studies were classed as "good" if answered yes to 9-11 of the CASP criteria and "satisfactory" if answered yes to 6-8 questions.

\section{Data extraction}

Data extraction was conducted in duplicate and inputted into a pilot spreadsheet according to outcomes measured; pain, knee stability, knee function, vascularization, inflammation and ligamentization. The authors, date, year of publication, PRP collection and application method, outcome measured, method of analysis, participant characteristics and results were recorded. Inconsistences were resolved via discussion between authors.

\section{Analysis}

A meta-analysis was considered for pain and knee function outcomes. Given comparable quantitative data, analysis would have involved using a fixed-effects model to calculate Relative risk (RR) and 95\% confidence intervals. Following this, a funnel plot and $I^{2}$ statistics would have been used to evaluate statistical heterogeneity. Review Manager V.5.3 would have then been utilised to create Forrest plots to summarise meta-analysis results. Where meta-analysis was not possible, a narrative analysis was conducted for each.

\section{Results}

The search identified 212 studies, reduced to 156 after de-duplication. Following title and abstract screening, 126 studies were found not to be investigating ACL reconstruction leaving 30 studies. The full text was reviewed against the inclusion and exclusion criteria resulting in 14 being removed due to not being RCTs, 1 study was not published in English and 2 studies were removed as they did not discuss our outcomes. This left 13 studies and is 




Fig. 1 PRISMA flow diagram. Systematic search process and results of the strategy showing number of papers found, removed during screening with reasons and final number of included studies

summarised in Fig. 1. The remaining studies underwent critical appraisal (Table 2). Studies were grouped into overarching healing or clinical themes before export into a data extraction table. Outcomes were grouped as follows: vascularization, inflammation, ligamentization, pain, knee stability and knee function. Methods of PRP collection and application were summarised. A summary of paper characteristics and results is presented in Table 1.

\section{Quality assessment}

CASP appraisal of methodology involved consideration of blinding protocols, demographical analysis between groups and standardisation of care amongst groups. There were a number of studies displaying deficits in aspects of their methodology. Four studies: Cerevellin et al. [11], 


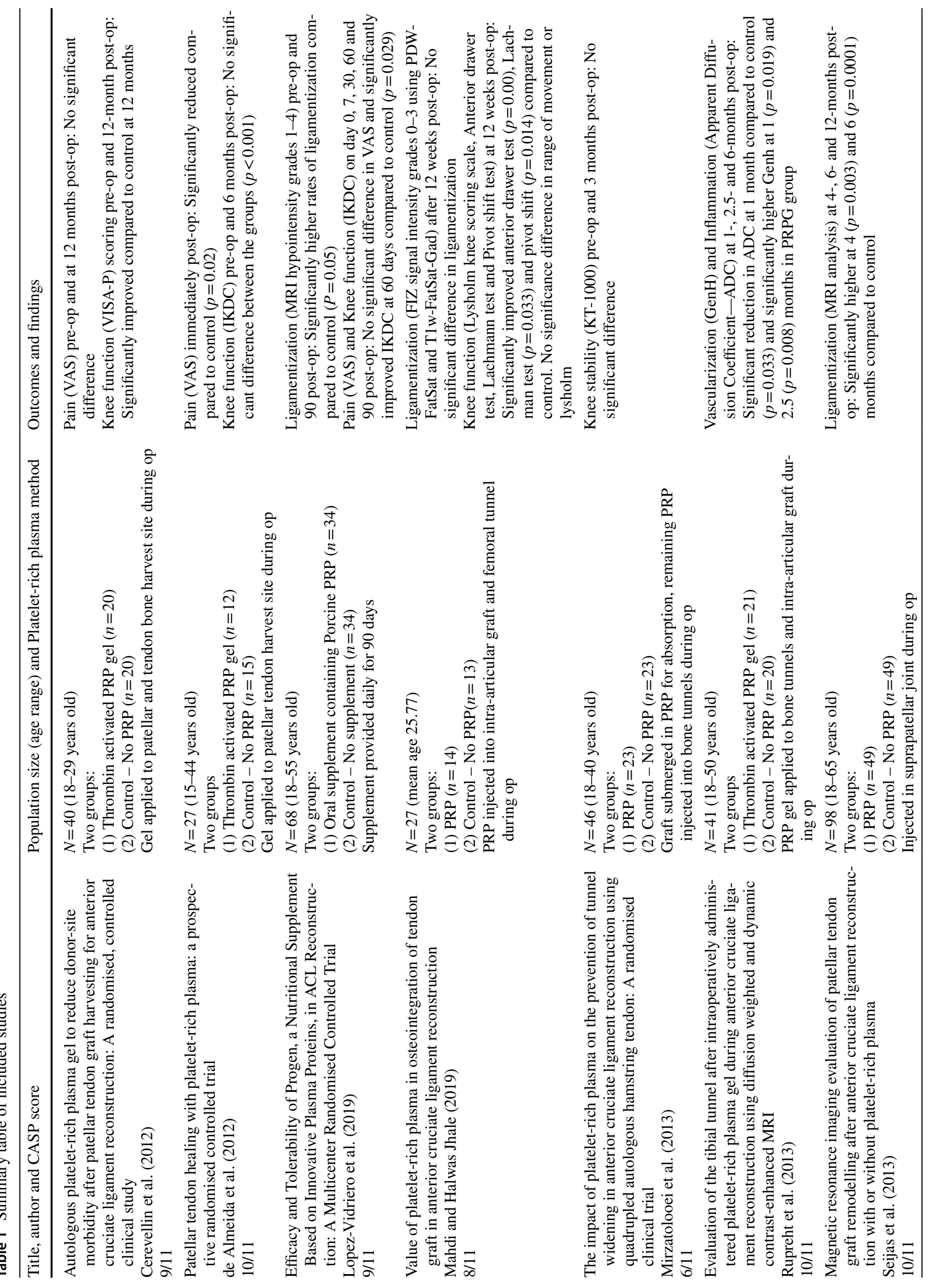




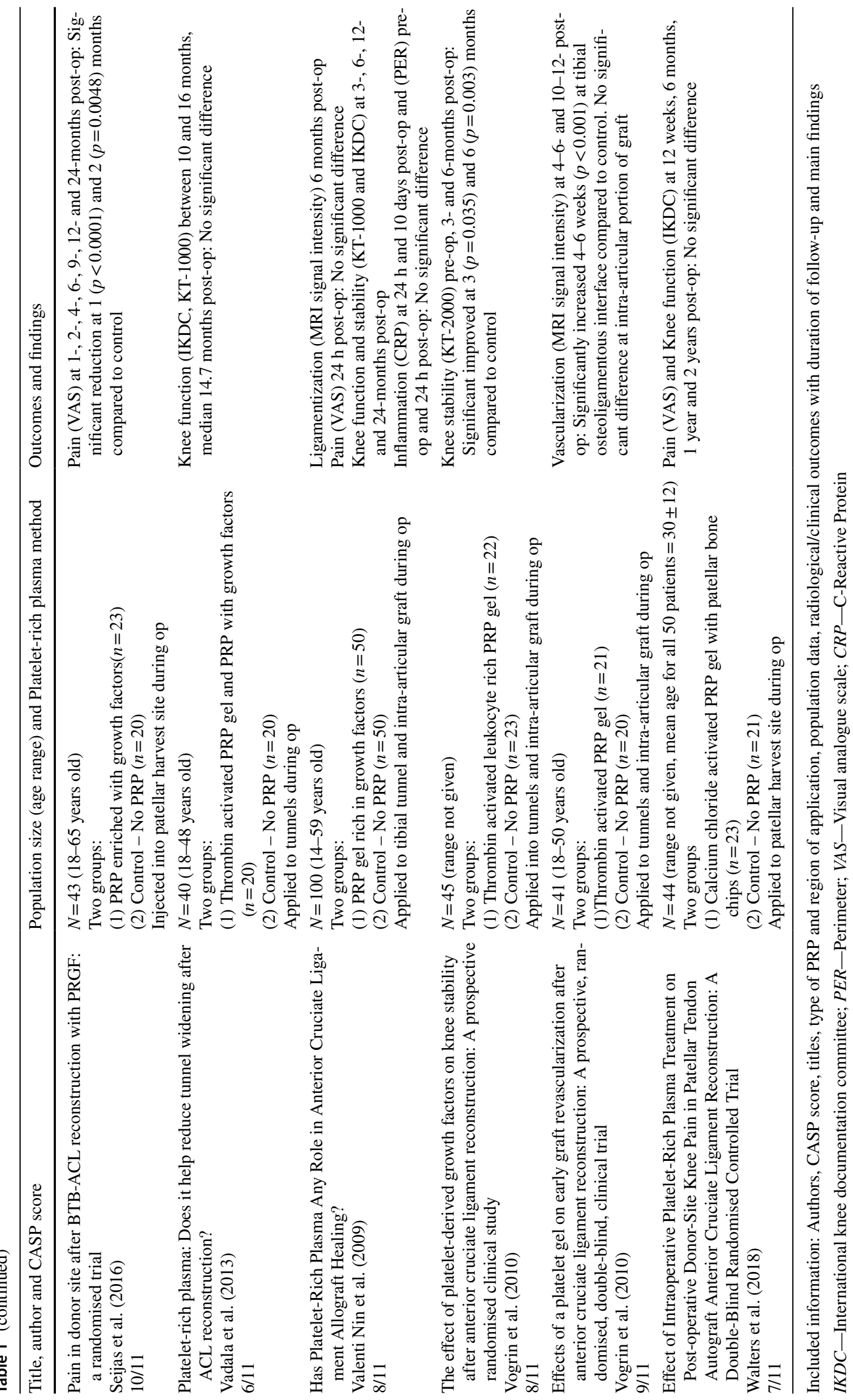


Lopez-Vidriero et al. [12], Mirzatolooei et al. [13] and Vogrin et al. [14] did not blind all participants and study personnel to treatment. A further three studies; de Almeida et al. [15], Mahdi and Halwas Jhale [16] and Vadala et al. [17] did not state whether all study personnel and participants were blinded. Four studies: Mahdi and Halwas Jhale [16], Mirzatolooei et al. [13], Vadala et al. [17] and Walters et al. [18] made no reference to demographic analysis between groups thus it was not possible to determine whether the groups were similar at the start of the study. Only Walters et al. [18] did not make reference to postoperative protocols so it is impossible to say whether the groups were treated equally throughout the entire study.

When appraising data from the studies, only three: de Almeida et al. [15], Rupreht et al. [19] and Seijas et al. [20] provided confidence intervals meaning no precision of intervention effect was reported for the remaining studies. Determination of interventional benefit against cost or harms was not possible for eight studies. Mirzatolooei et al. [13], Rupreht et al. [19], Seijas et al. [20], Vogrin et al. [14], Vogrin et al. [21] and Walters et al. [18] did not state adverse outcomes or reference any costs. Vadala et al. [17] and Valenti Nin et al. [22] reported no difference in adverse outcomes between groups but made no references to cost of the intervention and reported no significant difference between groups in any of the outcome measures.

Application of findings to the relevant patient population was scrutinised whereby three studies: Mirzatolooei et al. [13], Vadala et al. [17] and Valenti Nin et al. [22] showed no significant difference between groups for any outcomes at any time, suggesting these interventions did not provide greater value than existing treatments. All studies except Walters et al. [18] had samples that were biased towards young, athletic males. Table 2 provides in depth critical analysis of the included studies using CASP RCT grading. Scores for each individual study are provided in Table 1.

\section{PRP collection and application methods}

Two studies; Vogrin et al. [21] and [14], used the same apparatus for collection. Ten studies isolated platelets via centrifugation (range: 5-15 min and 1500-3200 rpm) whilst de Almeida et al. [15] used filtration separation. Seijas et al. [28] did not state method of separation. Five studies recorded platelet levels within PRP; de Almeida et al. [15] $\left(1,185,166 / \mathrm{mm}^{3} \pm 404,472 / \mathrm{mm}^{3}\right)$, Vogrin et al. [14] (average: 962 G/l range: 552-1326), Valenti Nin et al. [22] (average: $\left.837 \times 10^{3} / \mathrm{mm}^{3}\right)$, Walters et al. [18] (2-3 $\times$ above baseline) and M. Mahdi and Halwas Jhale [16] (5.0-7.0 platelets per preparation). Lopez-Vidriero et al. [12] stated the contents of the supplement sachets; $2500 \mathrm{mg}$ of chondroitin sulphate, $300 \mathrm{mg}$ of porcine PRP, $50 \mathrm{mg}$ hyaluronic acid and $40 \mathrm{mg}$ Vitamin $\mathrm{C}$. The remaining studies did not report platelet levels within PRP.

Six studies; Valenti Nin et al. [22], Rupreht et al. [19], Cerevellin et al. [11], de Almeida et al. [15], Vogrin et al. [21] and Mahdi and Halwas Jhale [16] employed methods to maximise PRP retention at application site. The methods used were peritendon and fat pad sutures, suturing PRP into the internal aspect of graft and no use of arthroscopic fluid. Only Lopez-Vidriero et al. [12] trialled multiple applications of intervention, with oral supplementation provided once daily for a 90-day period (Table 3).

\section{Clinical outcomes}

\section{Pain}

Six studies $(n=378)$ : Cerevellin,et al. [11], de Almeida, et al. [15], Lopez-Vidriero et al. [12], Seijas et al. [20], Valenti Nin et al. [22] and Walters et al. [18] measured the effect of PRP application on pain during Activities of daily living (ADLs). All studies reported a mean patient Visual analogue scale (VAS) [23] scored out of ten with ten being the highest level of pain. Significance was set at $p<0.05$ except LopezVidriero et al. [12] who used $p \leq 0.05$.

Two studies found that PRP caused a significant reduction in pain. De Almeida et al. [15] $(n=27)$ found that PRP significantly reduced pain immediately post-op with $3.8 \pm 1.0( \pm \mathrm{SD})$ compared to $5.1 \pm 1.4$ for the control $(p=0.02)$. Similarly, Seijas et al. [20] $(n=43)$, who measured up to 24 months post-op, observed significantly reduced pain at 1 month with 0.63 compared to 2.58 for the control $(p<0.0001)$ and 2 months with 0.54 compared to 2.21 for the control $(p=0.0048)$. The remaining four studies, of which the maximum period of follow-up was 2 years, reported no significant difference in pain at any interval.

\section{Knee stability}

Five studies $(n=258)$ : Mahdi and Halwas Jhale [16], Mirzatolooei et al. [13], Vadala et al. [17], Valenti Nin et al. [22] and Vogrin et al. [14] measured the effect of PRP on knee stability. Mirzatolooei et al. [13], Vadala et al. [17] and Valenti Nin et al. [22] used KT-1000 arthrometers whilst Vogrin et al. [14] used a KT-2000 arthrometer. Mahdi and Halwas Jhale [16] used Lachman's test [24]. Significance was set at $p<0.05$.

Two studies found that PRP significantly improved knee stability. Vogrin et al. [14] $(n=45)$, who observed up to 6-months post-op, found significantly improved knee stability at 3 -months post-op with a $4.9 \pm 1.8 \mathrm{~mm}$ displacement compared to $6.1 \pm 2.1 \mathrm{~mm}$ for the control $(p=0.035)$. This 
was also found at 6-months post-op with $4.7 \pm 1.9 \mathrm{~mm}$ compared to $6.7 \pm 2.1$ for the control $(p=0.003)$. Additionally, M. Mahdi and Halwas Jhale [16] $(n=27)$ found significantly reduced laxity at 12 weeks with $12 / 14$ participants having $\leq 5 \mathrm{~mm}$ displacement compared to $6 / 13$ for the control $(p=0.033)$. The remaining three studies, with a maximum follow-up of 2 years, showed no significant improvement in knee stability with the application of PRP.

\section{Knee function}

Seven studies $(n=364)$ : Cerevellin et al. [11], de Almeida et al. [15], Lopez-Vidriero et al. [12], Mahdi and Halwas Jhale [16], Vadala et al. [17], Valenti Nin et al. [22] and Walters et al. [18] evaluated the effect of PRP on knee function. All studies analysed knee function via IKDC questionnaires [25], except Mahdi and Halwas Jhale [16], who used Lysholm scores [26] and Cerevellin et al. [11] who used a Patellar tendon Victorian Institute of Sport Assessment questionnaire [27]. Significance was set at $P<0.05$ for all studies except Lopez-Vidriero et al. [12] who set significance at $P \leq 0.05$.

Two studies observed significantly improved knee function with PRP use. Lopez-Vidriero et al. [12] $(n=68)$, observing up to 90 days post-op, reported a significant improvement at 60 days with $62.5 \pm 11.7$ compared to $55.5 \pm 11.1$ for the control $(P=0.029)$. Further to this, Cerevellin et al. [11] $(n=40)$ measured up to 12 months postop and found significantly better scores at 12 months with $97.8 \pm 2.5( \pm \mathrm{SD})$ compared to $84.5 \pm 11.8$ for the control $(p=0.041)$. The remaining five studies, with a maximum follow-up of 2 years, found PRP had no significant effect on knee function at any time.

\section{Parameters of healing}

\section{Vascularization and cellularity}

Three studies ( $n=109)$; Rupreht et al. [19], Mahdi and Halwas Jhale [16] and Vogrin et al. [21] investigated the effect of PRP application on vascularisation of different parts of the ligament graft. Rupreht et al. [19] investigated the tibial tunnel using a 1.5 T MRI scanner to assess contrast enhancement gradient $\left(\mathrm{G}_{\mathrm{enh}}\right)$. Vogrin et al. [21] investigated the tibial osteoligamentous interface and intra-articular graft using contrast-enhanced MRI signal intensity. Mahdi and Halwas Jhale [16] used T1W-FatSat-Gad to assess vascularisation and PDW-Fat-Sat-signal grades for cellularity at the site of osteoligamentous integration (fibrous interzone-FIZ) in the femoral tunnel. Significance was set at $P<0.05$.
Two studies observed significantly increased levels of vascularisation with PRP administration. Rupreht et al. [19] $(n=41)$ measured up to 6-months post-op. They reported significantly increased vascularization at 1 month with a mean of 2.07 compared to 1.41 for the control $(p=0.019)$ and at 2.5 months, with a mean of 1.64 compared to 1.15 for the control $(p=0.008)$. Vogrin et al. [21] $(n=41)$ measured up to 12-weeks post-op and found significantly increased vascularization of the osteoligamentous interface at weeks $4-6$ with $0.33 \pm 0.09$ vs. $0.16 \pm 0.009$ for the control $(p<0.001)$.

Mahdi and Halwas Jhale [16] $(n=27)$, who had a minimum follow-up of 12 weeks, found PRP had no significant effect on vascularisation or cellularity at the femoral FIZ.

\section{Inflammation}

Two studies ( $n=141)$ : Rupreht et al. [19] and Valenti Nin et al. [22] analysed inflammatory parameters after PRP application. Valenti Nin et al. [22] measured C-reactive protein (CRP) and perimeter (PER) of the knee joint $(\mathrm{PER} 1=$ patella centre, $\mathrm{PER} 2=5 \mathrm{~cm}$ above superior patella edge). Rupreht, tal. [19] measured via Apparent diffusion coefficient (ADC), where oedema produced higher values. Significance was set at $P<0.05$.

Rupreht et al. [19] ( $n=41)$ measured up to 6-months post-op and reported significantly reduced ADC values at 1 -month post-op with $1.41(1 \pm 0.1)$ compared to 1.5 (1 $\pm 0.09)$ for the control $(p=0.033)$.

Valenti Nin et al. [22] $(n=100)$, found no significant difference in CRP up to 10 days post-op and PER $24 \mathrm{~h}$ post-op between groups.

\section{Ligamentization}

Three studies $(n=266)$ : Lopez-Vidriero et al. [12], Seijas et al. [28] and Valenti Nin et al. [22] investigated the effect of PRP on graft remodelling to determine rate of ligamentization via MRI signal intensity. Significance was set at $P<0.05$ except for Lopez-Vidriero et al. [12] who used $P \leq 0.05$.

Two papers found that PRP significantly increased the rate of ligamentization. Seijas et al. [28] $(n=98)$, measuring up to 12-months post-op, showed significantly increased remodelling at 4-months with 39 participants reaching moderately hyperintense as opposed to 23 for the control $(p=0.003)$. This was also seen at 6- months with 46 reaching moderately hyperintense or higher compared to 32 for the control $(p=0.0001)$. Lopez-Vidriero et al. [12] $(n=68)$ measured pre-op and 90 post-op and displayed significantly improved graft maturation at 90 days with 21 participants 
attaining grade 3 or higher as opposed to 13 for the control $(p=0.05)$.

Valenti Nin et al. [22] $(n=100)$ found no significant difference in ligamentization with PRP application at 6 months post-op.

\section{Discussion}

Success of ACL reconstructive surgery can be measured via clinical features or healing parameters. Literature spanning over a decade has offered insight into whether the use of biological augmentation can enhance these outcomes. These were first described in animal models, where PRP appeared to stimulate the healing processes. In human trials, research into the effects of PRP administration has been conducted in many clinical contexts, with varied results. Following surgery, patients are most concerned with pain levels and knee functionality; hence these outcomes have been widely investigated in the literature. Radiological outcomes provide another measure, allowing for a more rounded and comprehensive analysis of the effect of PRP. This systematic review aimed to collate the clinical and radiological results of RCTs to evaluate whether PRP would benefit those undergoing ACL reconstruction.

Of the clinical outcomes chosen for evaluation: pain, knee stability and function were most widely reported on, encompassing nine of the 13 included RCTs; with radiological considerations in six. Six studies [11, 12, 15, 18, 20, 22] evaluated pain, two of which found PRP reduced VAS scores in the early period post-op [15, 20]. Five studies [13, 14, 16, 17, 22] evaluated knee stability, two of which found PRP reduced anterior-posterior knee laxity $[14,16]$. No significant difference was found beyond 6 months. Seven studies [11, 12, 15-18, 22] evaluated knee function, two of which found PRP to improve overall knee function [11, 12]. No significant difference was found beyond 12 months. Three studies $[16,19,21]$ evaluated vascularization and cellularity, two of which found PRP to increase rate of vascularization [19, 21]. No significant difference was found beyond 12 weeks. PRP was not found to cause significant change in cellularity levels. Two studies [19, 22] measured the effect of PRP on inflammatory parameters, with only one showing a significant reduction in inflammation [19]. Three studies [12, 22, 28] evaluated ligamentization, two of which found PRP to increase rate of ligamentization $[12,28]$. No significant difference was found beyond 6 months.

The inflammatory response following injury or surgery leads to pain, release of inflammatory cytokines and oedema [29]. Pain is subjective, making it a difficult parameter to monitor in a standardised manner. VAS provides some mediation but cannot negate variation in individual pain thresholds. As pain influences ability to carry out rehabilitation protocols [30], it becomes a barrier to full recovery. Reductions in pain would enable improved proprioceptive and strength rehabilitation, therefore potentially increasing the speed and success of recovery. PRP has been shown to reduce pain levels [31]. Of the six studies that measured VAS, de Almeida et al. [15] demonstrated a significant reduction pain at $24 \mathrm{~h}$ post-surgery concurring with the results of Seijas et al. [20] at 1- and 2-months post-surgery. Although a number of the remaining studies suggested that PRP reduced pain, this failed to reach statistical significance hence it cannot be conclusively stated whether PRP has a significant effect on pain. As PRP has not been proven to influence pain, further research must be conducted to evaluate any analgesic role it may play.

Method of PRP collection may have an influence on its ability to exert influence on healing and hence clinical parameters. For the method of PRP collection, 10 papers $[11,13,14,16-21]$ used centrifugation whilst de Almeida et al. [15] used filtration. de Almeida et al. [15] produced significant results in VAS whilst 3 of 4 using centrifugation showed no significant change. These different methods may have an effect on the efficacy of PRP however it is difficult to show as there is not enough data on the use of filtration. More studies using filtration should be conducted to ascertain whether this produces any significant change.

Inflammation has been shown to facilitate angiogenesis and production of hyper-vascular granulation tissue during healing [34]. However, inflammatory cytokines and oedema have also been reported to have adverse effects on recovery from ACL reconstruction. It has been shown that inflammatory cytokines can lead to atrophy of the surrounding muscles which adversely effects knee stability and function [32]. Previous studies, such as Anitua et al. [33], have suggested that PRP has an anti-inflammatory effect which could imply useful applications in ACL reconstruction. Of the two studies that measured inflammation, Valenti Nin et al. [22] found that PRP had no effect on CRP or knee swelling whilst Rupreht et al. [19] found significantly reduced knee oedema at 1-month post-op. These results appear contradictory, but this may be due to the different times at which the measurements were taken. Valenti Nin et al. [22] only observed up to 10 days post-op whereas Rupreht et al. [19] measured at 1-, 2.5- and 6-months. This could mean that the anti-inflammatory effects of PRP are delayed and hence were not picked up by Valenti Nin et al. [22].

The lack of significant results beyond one month could be due to PRP only having short-term effects or that the inflammatory phase is receding meaning tangible results will not be seen later in the studies. This is supported by Janssen and Scheffler [35], who state that the proliferative phase is over after 4-12 weeks. Alternatively, these effects could be dependent upon the composition of PRP. Azcarate et al. [36], who furthered the work of Valenti Nin et al. [22], added 
another intervention group; PRP without leukocytes. This form was found to significantly reduce CRP and swelling suggesting that it had more potent anti-inflammatory effects. This provides another avenue for research, suggesting that composition of PRP could alter its influence over different outcomes. As inflammation plays a key role in vascularisation and healing, the anti-inflammatory effects of PRP could be counterproductive. However, it is excessive inflammation that limits healing and therefore finding the balance between the angiogenic effects and limiting excessive inflammation could be integral for PRP to be beneficial in this area.

Further to this, it has been reported that platelet concentrations should be over $1 \times 10^{6} / \mathrm{ml}$ [37]; roughly 5 times baseline (whole blood $2 \times 10^{5} / \mathrm{ml}$ ) for PRP to be effective. Five studies [14-16, 18, 22] reported platelet content within PRP concentrations with only de Almeida et al. [15] indicating an average above $1 \times 10^{6} / \mathrm{ml}$. This may explain the significant reduction in VAS reported by de Almeida et al. [15] immediately post-op compared to the lack of a significant difference found by Valenti Nin et al. [22] $24 \mathrm{~h}$ post-op, who reported an average platelet concentration of $837 \times 10^{3} / \mathrm{mm}^{3}$. As the remaining seven studies did not record platelet levels, it is impossible to investigate whether their insignificant results could be attributed to using PRP with insufficient platelet concentrations. Further studies should be completed to investigate how platelet concentrations can influence level of clinical benefit within this setting.

Vascularisation is essential for conversion to a functional ligament. In the bone tunnels this aids osteointegration, which roots the ligament providing a stable attachment. In the intra-articular portion, this converts the tendinous structure to resemble the native ACL. Upon grafting, the tendon tissue undergoes remodelling to acquire ligament characteristics, such as higher levels of irregular collagen and proteoglycan bundles that remodel to produce a densely packed, parallel, uniform morphology [38]. Therefore, ligamentization is essential for the graft to achieve the strength and durability required to fulfil the role of the ACL. Hence, the rate of vascularisation and ligamentization have a direct impact on recovery time, improving knee stability and function. This could benefit those looking for a quicker recovery, such as high-level athletes or those who cannot afford time away from work to recover. Studies of the angiogenic effects of PRP have produced positive results, such as those investigating neovascularisation in cardiac muscle [39], suggesting PRP has potential to improve vascularisation in our setting.

Rupreht et al. [19] and Vogrin et al. [21] found no significant increase in intra-articular vascularisation. The lack of significant data could be attributed to a decreased retention of the PRP gel at the intra-articular portion of the graft, limiting its angiogenic influence. Significantly increased levels of bone tunnel vascularisation were observed at 4-6 weeks post-op, with no benefit seen beyond this point.
This is supported by the results of Silva and Sampaio [40] who observed no significant difference in vascularisation 3 -months post-op. This suggests that PRP is no longer able to influence proceedings. This could be a dose issue as only single applications of PRP are used in our studies with exception of Lopez-Vidriero et al. [12]. Alternatively, much like the effects seen in inflammatory outcomes, this may be due to a transient role of new vasculature. This is supported by Janssen and Scheffler [35] who state that vascularisation occurs in the proliferative phase (4-12 weeks) and once it has played its role it recedes. Therefore, our question becomes not just how much vascularisation is present, but how early it is occurring.

Of the 12 studies that surgically administered PRP, seven $[11,15,16,19,21,22,28]$ described attempts at PRP retention at the site of application. Of these seven studies, all except Valenti Nin et al. [22] reported significant effects of PRP. In comparison, the five studies [13, 14, 17, 18, 20] that did not attempt to retain PRP, three $[13,17,18]$ did not report significant effects. This discrepancy may be owed to the time in which PRP is retained at the site, therefore influencing the ability to yield healing benefits. A comparison of effects of PRP with and without retention efforts, could inform on the significance of this factor.

Following vascularisation, ligamentization occurs at 12 weeks and is continuous throughout recovery [41]. Faster ligamentization means that the graft will be able to function as an ACL earlier, decreasing the time taken to transition through the rehabilitation programme. Lopez-Vidriero et al. [12] demonstrated early benefits in ligamentization at 90-days post-op, whilst Seijas et al. [28] demonstrated a more prolonged benefit with significantly higher levels at both 4 and 6 months. This suggests PRP increases the early rate of ligamentization. However, results from Valenti Nin et al. [22] showed no significant difference at the 6-month mark; congruent with later research by Azcarate et al. [36].

Whilst the results of Lopez-Vidriero et al. [12] and Seijas et al. [28] are in agreement, it should be highlighted that the comparison of these results proves difficult due to different forms of PRP being used; Lopez-Vidriero et al. [12] provided oral supplementation of PRP, Seijas et al. [28] using simple injectable PRP and Valenti Nin et al. [22] using PDGF gel. On the other hand, the use of different preparations of PRP may help in determining which form is most suited in improving ligamentization rates. In addition, only Lopez-Vidriero, E., et al. (2019) provided more than one instance of application. This study provided significant improvement in knee function and ligamentization which could suggest repeat applications provides more substantial benefit. Unlike inflammation and vascularisation, ligamentization has been found to continue well beyond 6 months, and the lack of significant data beyond this stage could suggest a dose related issue. Repeat applications of PRP gel should 
be conducted to determine whether this could augment its influence, or further investigation into the oral supplement should be conducted to determine whether this is a superior form and whether specific preparations of PRP are best suited for this parameter.

The ultimate goal of reconstructing the ACL is to restore stability and function of the knee. However, studies suggest that of those with ACL injuries, only 55\% returned to their competitive sport of choice [42]. Success of the surgery depends on a combination of ability to take part in rehabilitation, effected by pain and inflammation, and rate of graft remodelling based on vascularization and ligamentization. Stability is a measure of the ability of the ACL to limit anterior-posterior translocation of the tibia in relation to the femur. Joint laxity has been associated with dysfunctional and injury-prone ACLs [43], hence a stable joint is key to recovery and return to sports. Vogrin [14] showed reduced laxity at 3 and 6 months using KT-2000 arthrometers whilst M. Mahdi and Halwas Jhale [16] also showed significantly reduced laxity at 12 weeks post-op via use of the Lachman's test. The remaining three studies found no significant difference in stability when measuring using KT-1000 arthrometers. The lack of consistent, statistically significant differences between PRP and control groups means that it cannot be stated that PRP exerts influence over the anterior-posterior laxity of the knee joint. The contrasting results provide an avenue for further research into the influence of PRP on anterior laxity, as an improvement in this outcome could be directly beneficial to those undergoing ACL reconstruction.

On the other hand, knee function provides the yardstick by which all patients will measure the success of their procedure. Thus, demonstration of a tangible difference supplied by PRP is a key outcome. Generally, no significant improvement in IKDC scores was seen when compared to the control. However, Lopez-Vidriero et al. [12] did report a significant improvement compared to control at 60 days post-op, but not at any other date of investigation. In addition, Cerevellin et al. [11] recording significantly improved VISA-P scores at 12 months could indicate some influence on knee function post-op. However, with five of the seven studies showing no statistically significant difference in knee function between groups, this indicates PRP does not have a significant effect on knee function post-op. This being said, it is important to consider that the differing results may be due to the time intervals at which the IKDC questionnaires were completed. Lopez-Vidriero et al. [12] was the only study to record prior to 12 weeks post-op, doing so on three occasions. The significant result of Lopez-Vidriero et al. [12] resides early in the recovery process, indicating that PRP may have an effect, but wears off by later stages. Hence, it would not have been observed at the time intervals used by the other studies. This early influence mirrors the effects demonstrated in vascularisation, inflammation and ligamentization.

Whilst a strong relationship is not established between PRP and improved knee function, there is room for further investigation in this area. Frequency of PRP applications may offer an interesting addition to these experiments, determining whether a single application is limiting its effect. This may explain why the benefits are only seen early in the study and is supported by Tavassoli et al. [44] who showed the efficacy of PRP increased after multiple injections over time. In addition, whilst it may appear that earlier and more regular analysis of knee function would be an improvement for subsequent studies, the restrictive nature of the rehabilitation protocols [45] may limit the amount of knee function that can be evaluated in the earlier stages of healing.

\section{Limitations of study}

The strengths of this study include the use of RCTs, so the data collected are of high quality. The use of three major e-databases ensured much of the applicable literature was found via our search. Two authors independently performed the search and critically appraised the included studies in detail using the CASP criteria limiting individual error.

Comparison between studies proved to be difficult due to the varying methods of PRP application. There appears to be no standardised approach, meaning caution must be taken when comparing results. We suggest a standardised protocol for the collection and application of PRP with assessment of platelet concentration would make inter-study comparison more reliable.

In addition, measurement intervals were inconsistent amongst the studies. As most of PRP's significant impacts were early on in the healing process, some studies may therefore miss the intervention effect due to prolonged periods between measurement. Using published data on when processes occur, e.g., vascularization, may inform on when data collection should be conducted.

This study aimed to determine PRP effects following ACL reconstruction for application to the wider population. Unfortunately, the literature is heavily biased towards a younger male athletic populous. Whilst it has been established that the incidence of ACL rupture is higher in the athletic population than the general population [46] it has also been shown that females have a 1.5 times greater risk of rupture when compared to male athletes [47]. Therefore, even though the athletic component of the distribution is representative of ACL injury distribution, the lack of female representation makes the results less applicable as a result of potential selection bias. Future research may benefit from incorporating female participants at a higher frequency. 
The detailed critical appraisals (Table 2) show further biases of the studies, including the lack of information regarding blinding and demographic data. The specification of "English language" in our inclusion criteria has exposed our study to selection bias. The lack of research into unpublished work and exclusive use of electronic databases will have increased exposure to publication bias.

\section{Conclusions}

PRP appears to exert early influence on healing in the form of vascularisation and granulation tissue formation, culminating in higher rates of ligamentization. Reductions in swelling and CRP levels suggest PRP could be of benefit in the acute stages of recovery. However, long-term effects were not demonstrated suggesting the influence of PRP to be limited. With no consensus reached on the impact of PRP on pain, knee stability and resultant knee function, future research on these areas must be conducted before a conclusion can be made. Future research may benefit from standardising PRP, incorporation of multiple doses, measurement of platelet levels and increased frequency of observation. Alternatively, more comprehensive comparison between different forms of PRP can indicate which is best for each outcome accordingly.

\section{Appendix}

See Figs. 2, 3, 4, Tables 2 and 3.
Fig. 2 Medline search strategy. All search terms shown along with combinations of terms using "and" or "or" function followed by number of results at each stage
Database: Ovid MEDLINE(R) <1946 to June Week 5 2021>, Ovid MEDLINE(R) Daily Update $<$ July 09, 2021>

Search Strategy:

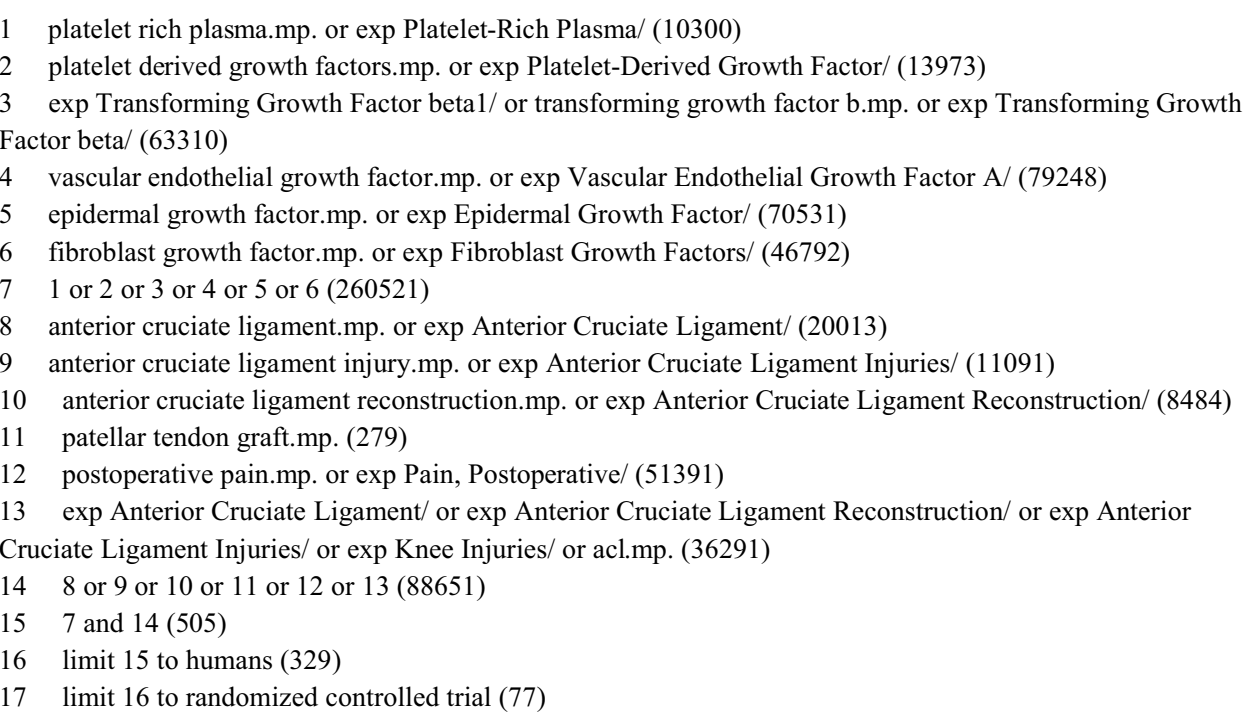


Fig. 3 Embase search strategy. All search terms shown along with combinations of terms using "and" or "or" function followed by number of results at each stage
Database: Embase Classic+Embase $<1947$ to 2021 July 09>

Search Strategy:

1 platelet rich plasma.mp. or exp Platelet-Rich Plasma/ (19692)

2 platelet derived growth factors.mp. or exp Platelet-Derived Growth Factor/ (25328)

3 exp Transforming Growth Factor beta1/ or transforming growth factor b.mp. or exp Transforming Growth

Factor beta/ (136442)

4 vascular endothelial growth factor.mp. or exp Vascular Endothelial Growth Factor A/ (95438)

5 epidermal growth factor.mp. or exp Epidermal Growth Factor/ (206707)

6 fibroblast growth factor.mp. or exp Fibroblast Growth Factors/ (91259)

71 or 2 or 3 or 4 or 5 or $6(516707)$

anterior cruciate ligament.mp. or exp Anterior Cruciate Ligament/ (30655)

9 anterior cruciate ligament injury.mp. or exp Anterior Cruciate Ligament Injuries/ (11309)

10 anterior cruciate ligament reconstruction.mp. or exp Anterior Cruciate Ligament Reconstruction/ (14727)

11 patellar tendon graft.mp. (387)

12 postoperative pain.mp. or exp Pain, Postoperative/ (82195)

13 exp Anterior Cruciate Ligament/ or exp Anterior Cruciate Ligament Reconstruction/ or exp Anterior

Cruciate Ligament Injuries/ or exp Knee Injuries/ or acl.mp. (56246)

148 or 9 or 10 or 11 or 12 or 13 (139009)

157 and $14(1250)$

16 limit 15 to humans (895)

17 limit 16 to randomized controlled trial (113)

\begin{tabular}{|c|c|c|c|c|c|}
\hline$\# 27$ & 22 & $\begin{array}{l}\text { \#26 AND \#25 } \\
\text { Indexes }=S C 1 \text {-EXPANDED, SSCI, A\&HCI, CPCI-S, CPCI-SSH, ESCI Timespon=All years }\end{array}$ & $\# 14$ & 24,747 & $\begin{array}{l}\text { TS=(anterior cruciate ligament) } \\
\text { Indexes"SCI-EXPAVDED, SSCI, A\&HCI, CPCI-S, CPCI-SSH, ESCI Timespan=All years }\end{array}$ \\
\hline$\# 26$ & 446,392 & $\begin{array}{l}\text { TS=(randomised control trial) } \\
\text { Indexes }=S C I-E X P A N D E D, S S C I, A \& H C I, C P C I-S, C P C I-S S H, E S C I \text { Timespan=All years }\end{array}$ & $\# 13$ & 336,435 & 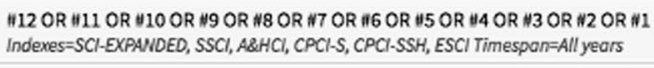 \\
\hline$\# 25$ & 294 & $\begin{array}{l}\text { \#24 AND \#23 } \\
\text { Indexes=SCI-EXPANDED, SSCI, A\&HCI, CPCI-S, CPCI-SSH, ESCI Timespon=All years }\end{array}$ & $\# 12$ & 95,197 & $\begin{array}{l}\text { TS=(fibroblast growth factors) } \\
\text { Indexes"SCI-EXPAMDED, SSCI, A\&HCI, CPCI-S, CPCI-SSH, ESCI Timespan=All years }\end{array}$ \\
\hline$\# 24$ & $4,156,225$ & $\begin{array}{l}\text { TS=(human) } \\
\text { Indexes=SCl-EXPANDED, SSCI, A\&HCI, CPCI-S, CPCI-SSH, ESCI Timespon=All years }\end{array}$ & $\# 11$ & 95,197 & $\begin{array}{l}\text { TS"(fibroblast growth factor) } \\
\text { Indexes=SCI-EXPANDED, SSCI, A\&HCI, CPCI-S, CPCI-SSH, ESCI Timespon=All years }\end{array}$ \\
\hline$\# 23$ & 1,303 & \#22 AND \#13 & $\# 10$ & 95,480 & $\begin{array}{l}\text { TS=(epidermal growth factor) } \\
\text { Indexes=SCI-EXPAVIDED, SSCI, A\&HCI, CPCI-S, CPCI-SSH, ESCI Timespan=All years }\end{array}$ \\
\hline$\# 22$ & 121,748 & CCI-EXPANDED, SSCI, A\&HCI, CPCI-S, CPCI-SSH, ESCI Timespar & \#9 & 77,705 & $\begin{array}{l}\text { TS=(vascular endothelial growth factor A) } \\
\text { Indexes=SCI-EXPANIDED, SSCI, A\&HCI, CPCI-S, CPCl-SSH, ESCI Timespon=All years }\end{array}$ \\
\hline \multirow[t]{2}{*}{$\# 21$} & 20,077 & CCI-EXPANDED, SSCI, A\&HCI, CPCI-S, CPCI-SSH, ESCI Timespon=All years & $\# 8$ & 89,551 & $\begin{array}{l}\text { TS=(vascular endothelial growth factor) } \\
\text { Indexes=SCI-EXPAMDED, SSCI, A\&HCI, CPCI-S, CPCI-SSH, ESCI Timespan=All years }\end{array}$ \\
\hline & & 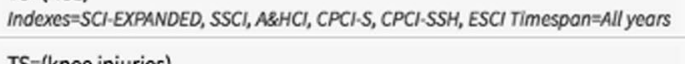 & $\# 7$ & 70,476 & $\begin{array}{l}\text { TS=(transforming growth factor beta) } \\
\text { Indexes=SCI-EXPANDED, SSCI, A\&HCI, CPCI-S, CPCl-SSH, ESCI Timespon=All years }\end{array}$ \\
\hline$\# 20$ & 52 & $\begin{array}{l}\text { TS=(knee injuries) } \\
\text { Indexes=SCI-EXPANDED, SSCI, A\&HCI, CPCI-S, CPCI-SSH, ESCI Timespon=All years }\end{array}$ & $\# 6$ & 13,683 & $\begin{array}{l}\text { TS=(transforming growth factor } \mathrm{b} \text { ) } \\
\text { Indexes=SCI-EXPANDED, SSCI, A\&HCI, CPCI-S, CPCl-SSH, ESCI Tìmespan=All years }\end{array}$ \\
\hline$\# 19$ & 71,292 & $\begin{array}{l}\text { TS=(postoperative pain) } \\
\text { Indexes=SCI-EXPANDED, SSCI, A\&HCI, CPCI-S, CPCI-SSH, ESCI Timespon=All years }\end{array}$ & $\# 5$ & 4,070 & $\begin{array}{l}\text { TS=(transforming growth factor beta1) } \\
\text { Indexcs=SCl-EXPANDED, SSCl, A\&HCl, CPCI-S, CPCI-SSH, ESCI Timespon=All years }\end{array}$ \\
\hline$\# 18$ & 3,510 & $\begin{array}{l}\text { TS=(patellar tendon graft) } \\
\text { Indexes=SCI-EXPANDED, SSCI, A\&HCI, CPCI-S, CPCI-SSH, ESCI Timespan=All years }\end{array}$ & $\# 4$ & 21,303 & $\begin{array}{l}\text { TS=(platelet-derived growth factor) } \\
\text { Indexes=SCI-EXPANDED, SSCI, A\&HCI, CPCI-S, CPCI-SSH, ESCI Timespon=All years }\end{array}$ \\
\hline$\# 17$ & 14,753 & $\begin{array}{l}\text { TS=(anterior cruciate ligament reconstruction) } \\
\text { Indexes=SCI-EXPANDED, SSCI, A\&HCI, CPCI-S, CPCI-SSH, ESCI Timespan=All years }\end{array}$ & $\# 3$ & 23,279 & $\begin{array}{l}\text { TS=(platelet derived grovath factors) } \\
\text { Indexes=SCI-EXPANDED, SSCl, } \mathrm{ARHCl}, \mathrm{CPCl}-\mathrm{S}, \mathrm{CPCl}-\mathrm{SSH}, \mathrm{ESCl} \text { Timespon=All yeors }\end{array}$ \\
\hline$\# 16$ & 11,848 & $\begin{array}{l}\text { TS=(anterior cruciate ligament injuries) } \\
\text { Indexes=SCI-EXPANDED, SSCI, A\&HCI, CPCI-S, CPCI-SSH, ESCI Timespan=All years }\end{array}$ & $\# 2$ & 13,084 & $\begin{array}{l}\text { TS=(platelet-rich plasma) } \\
\text { Indexes=SCI-EXPAVIDED, SSCI, A\&HCI, CPCI-S, CPCI-SSH, ESCI Tìmespon=All years }\end{array}$ \\
\hline$\# 15$ & 11,848 & $\begin{array}{l}\text { TS" =(anterior cruciate ligament injury) } \\
\text { Indexes=SCI-EXPANDED, SSCI, A\&HCI, CPCI-S, CPCI-SSH, ESCI Timespan=All years }\end{array}$ & $\# 1$ & 14,631 & $\begin{array}{l}\text { TS=(platelet rich plasma) } \\
\text { Indexes=SCI-EXPNWDED, SSCI, ARHCI, CPCI-S, CPCI-SSH, ESCI Timespon=All yeors }\end{array}$ \\
\hline
\end{tabular}

Fig. 4 Web of Science search strategy. All search terms shown along with combinations of terms using "and" or "or" function followed by number of results at each stage

Authors' contributions JM contributed to study conception, data collection, data analysis, drafted and reviewed final manuscript. KHK contributed to study conception, data collection, data analysis, drafted and reviewed final manuscript. IA drafted and reviewed final manuscript.
FD drafted and reviewed final manuscript. AM contributed to study conception, drafted and reviewed final manuscript.

Funding Not applicable. 


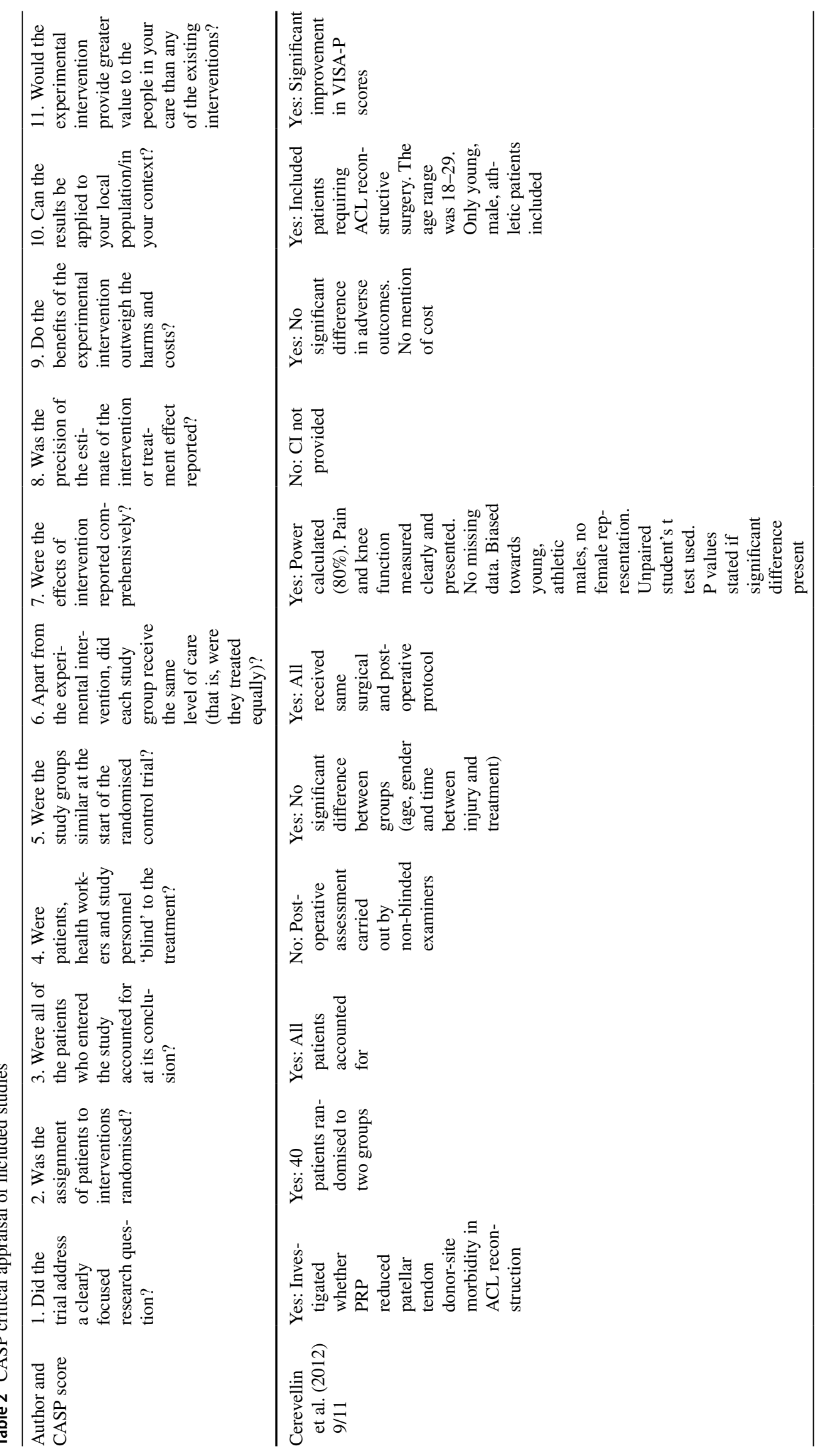




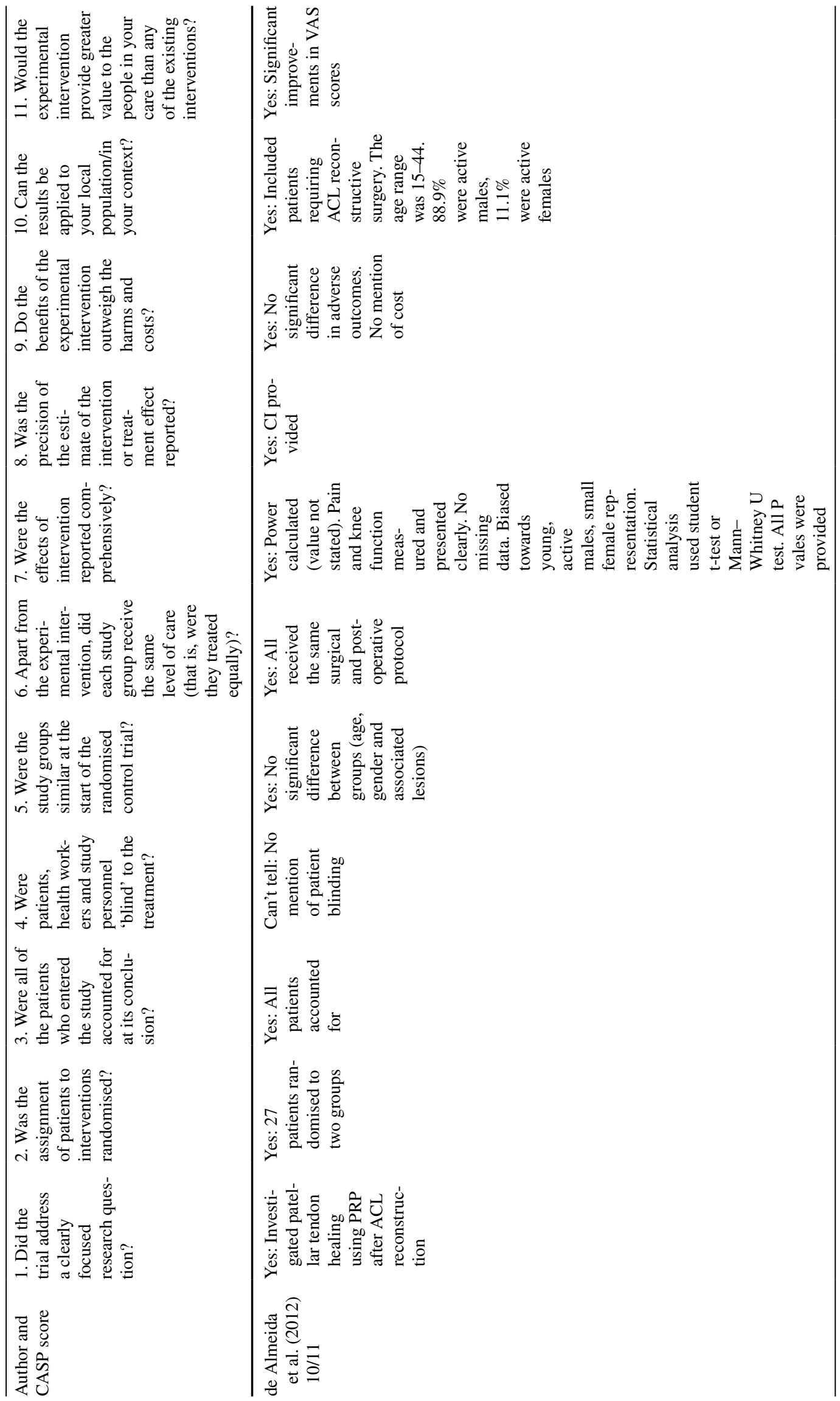




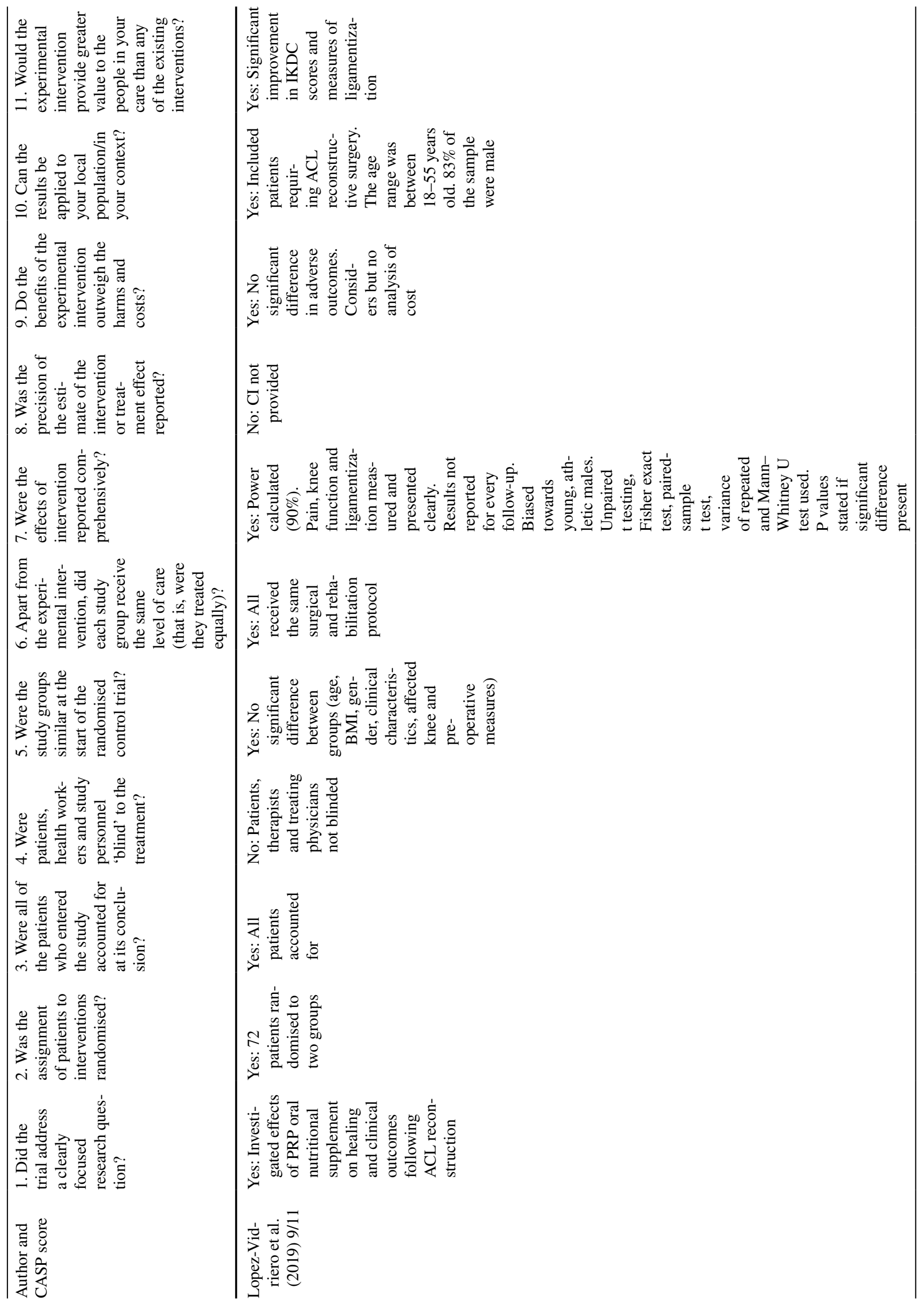




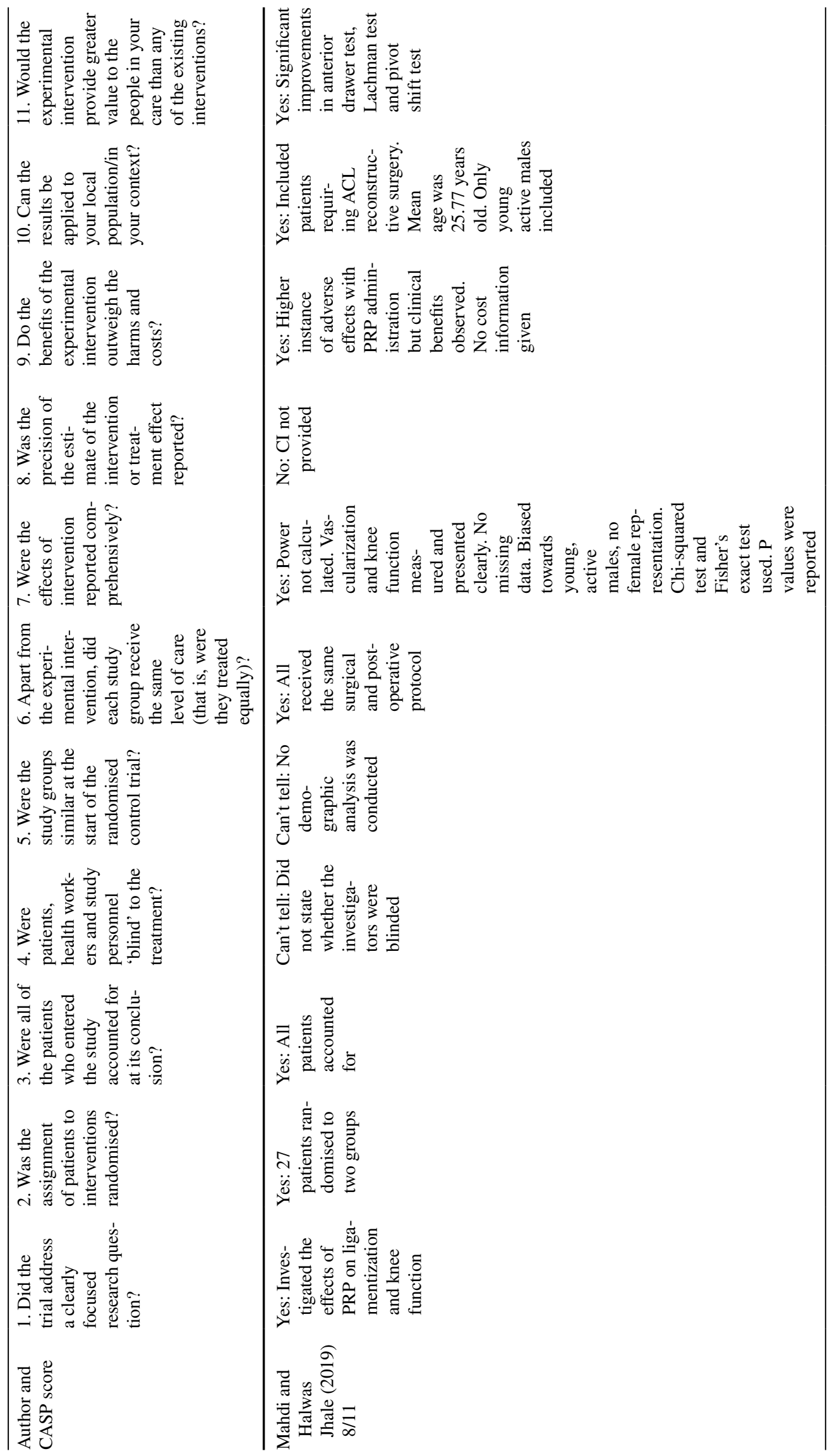




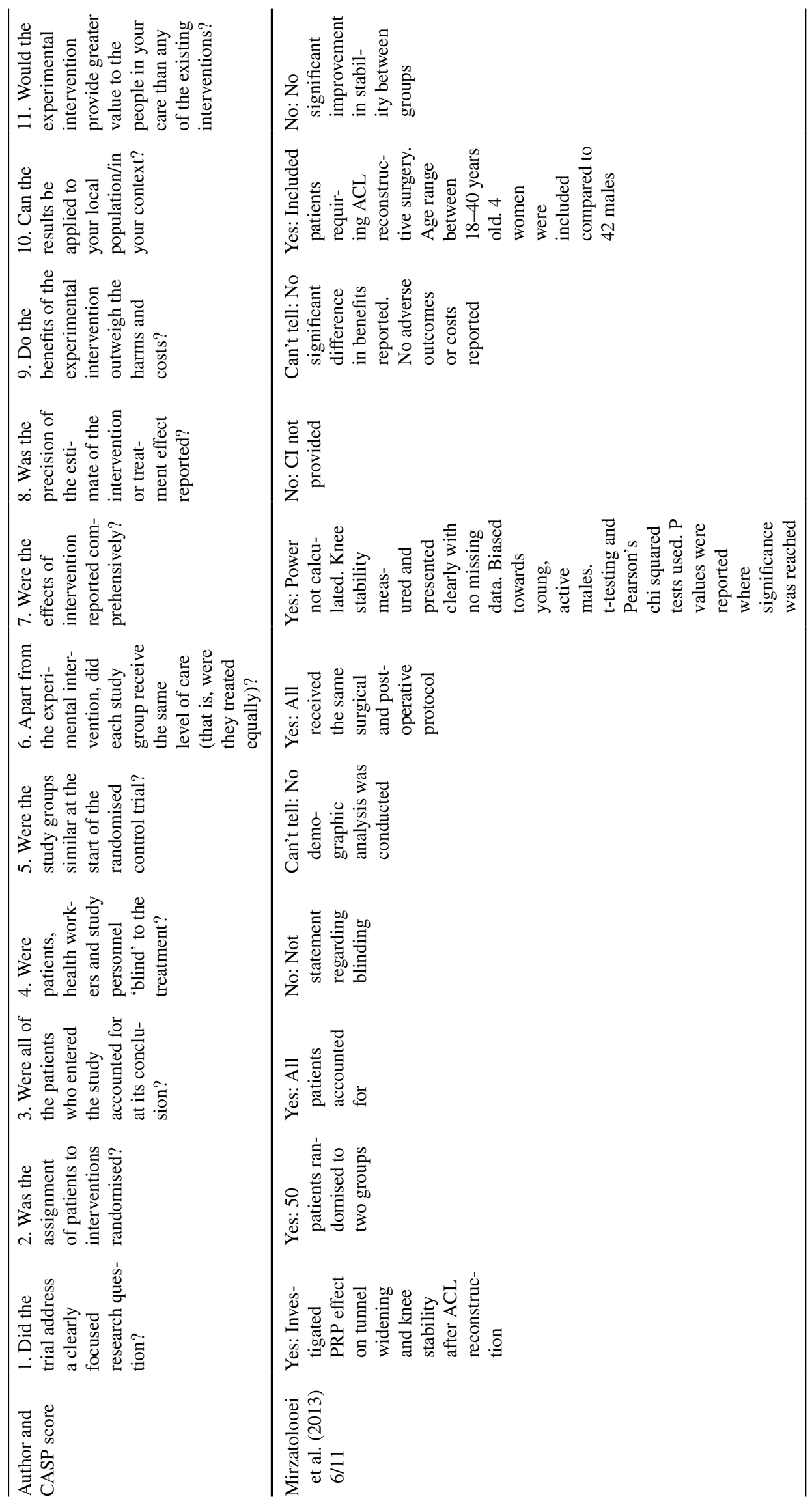




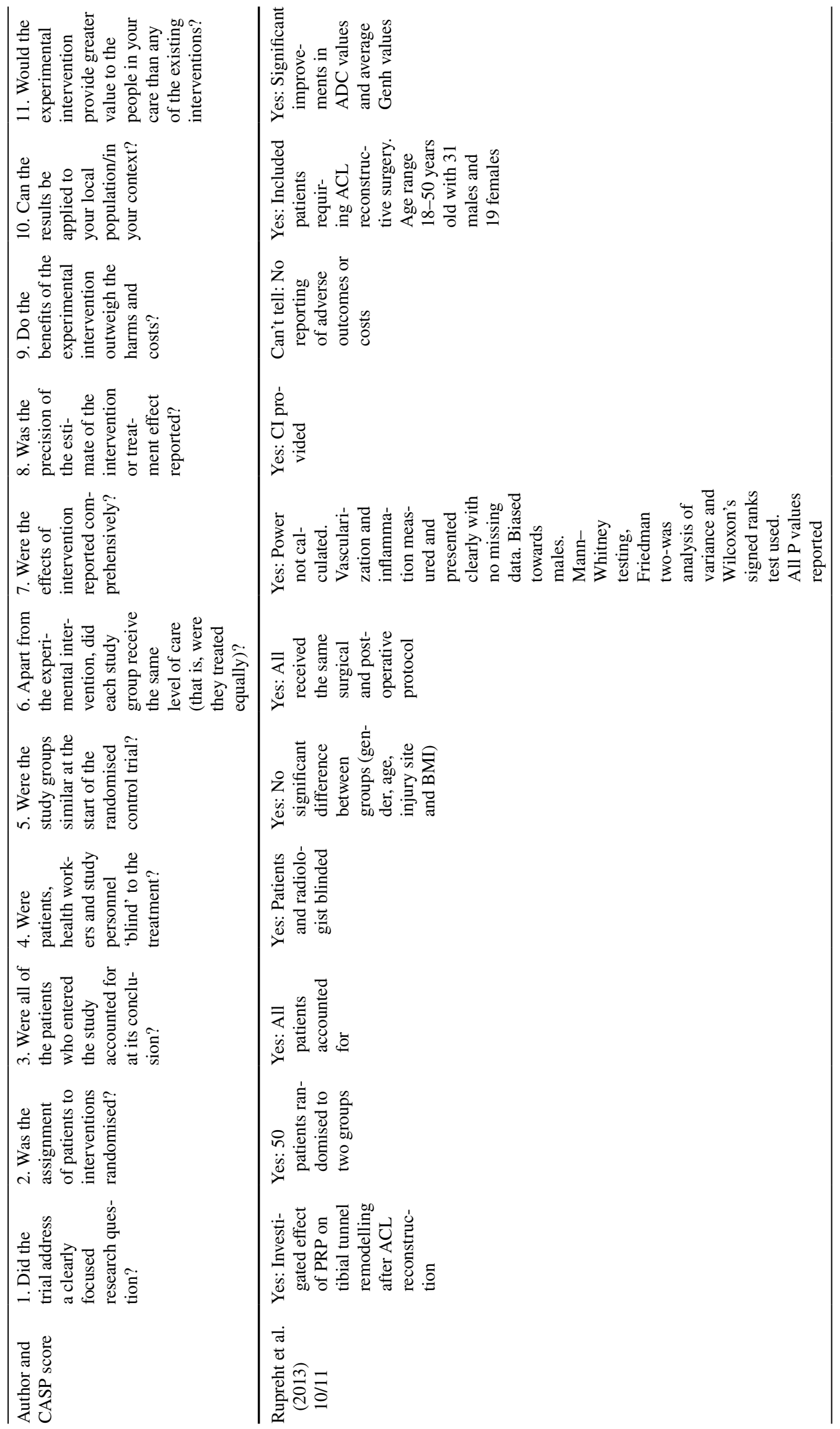




\begin{tabular}{|c|c|}
\hline 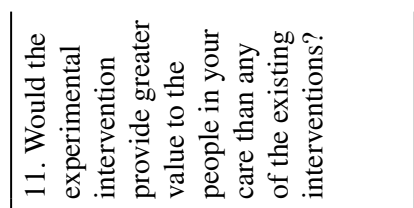 & 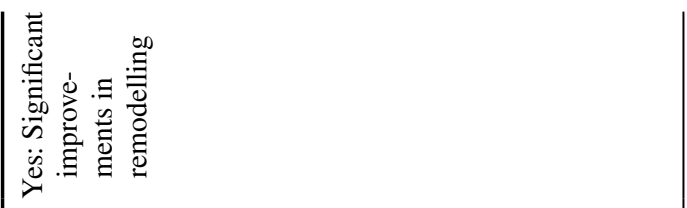 \\
\hline 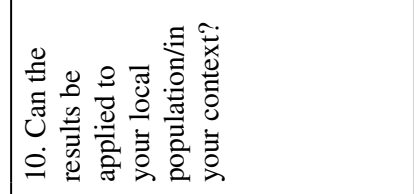 & 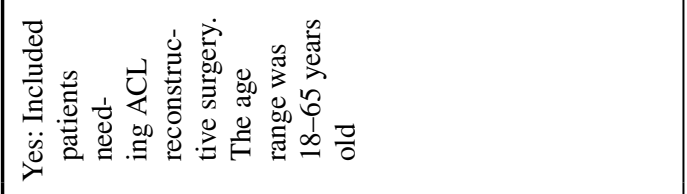 \\
\hline 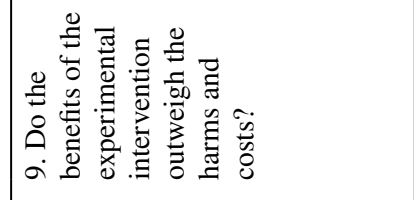 & 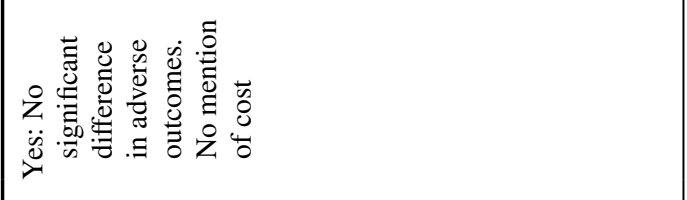 \\
\hline 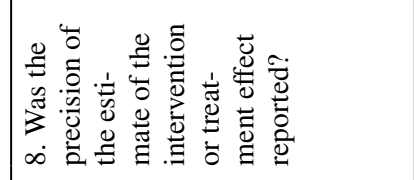 & 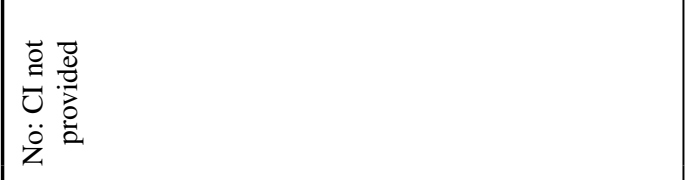 \\
\hline 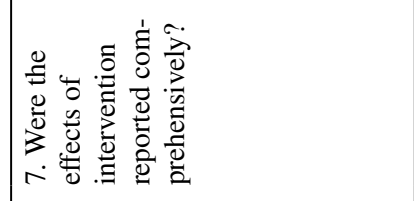 & 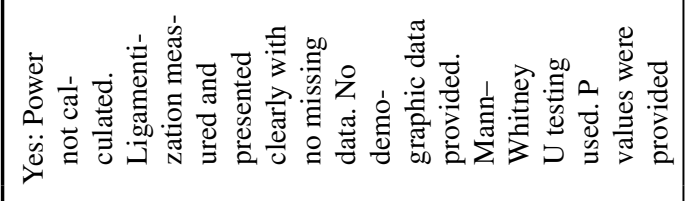 \\
\hline 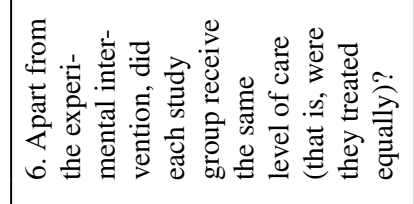 & 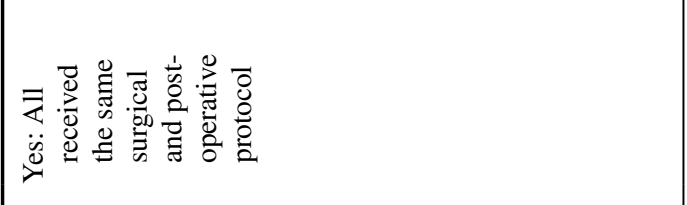 \\
\hline 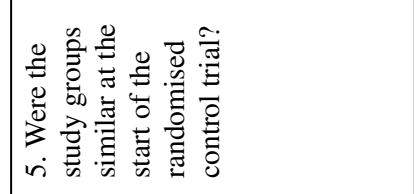 & 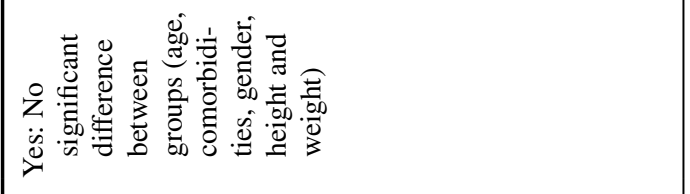 \\
\hline 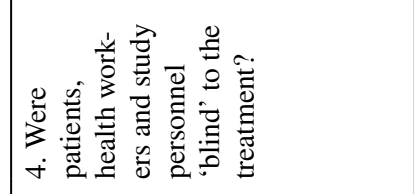 & 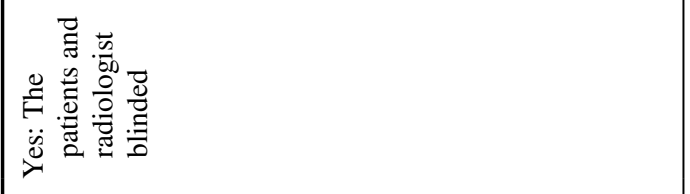 \\
\hline 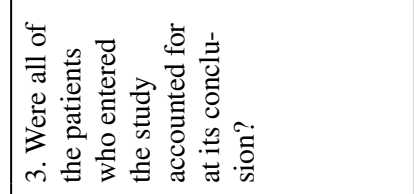 & 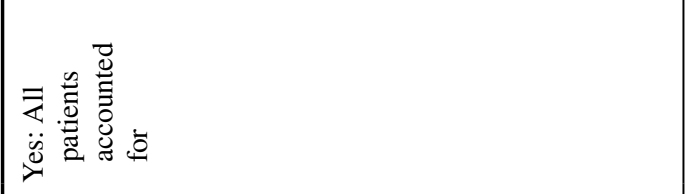 \\
\hline 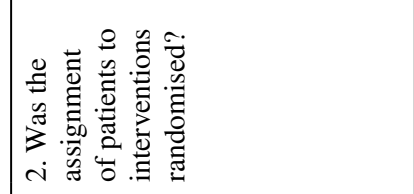 & 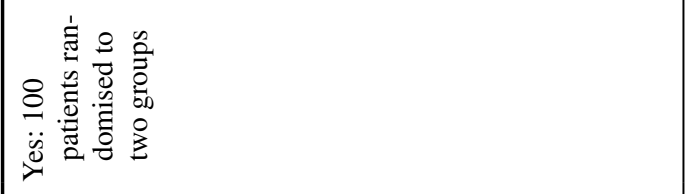 \\
\hline 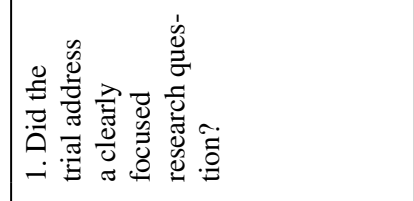 & 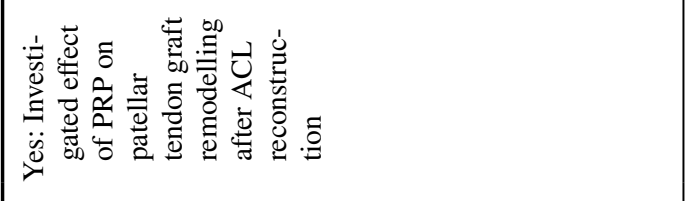 \\
\hline 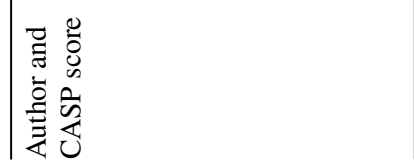 & 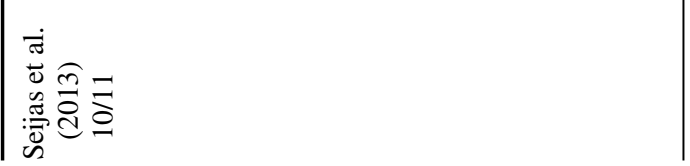 \\
\hline
\end{tabular}




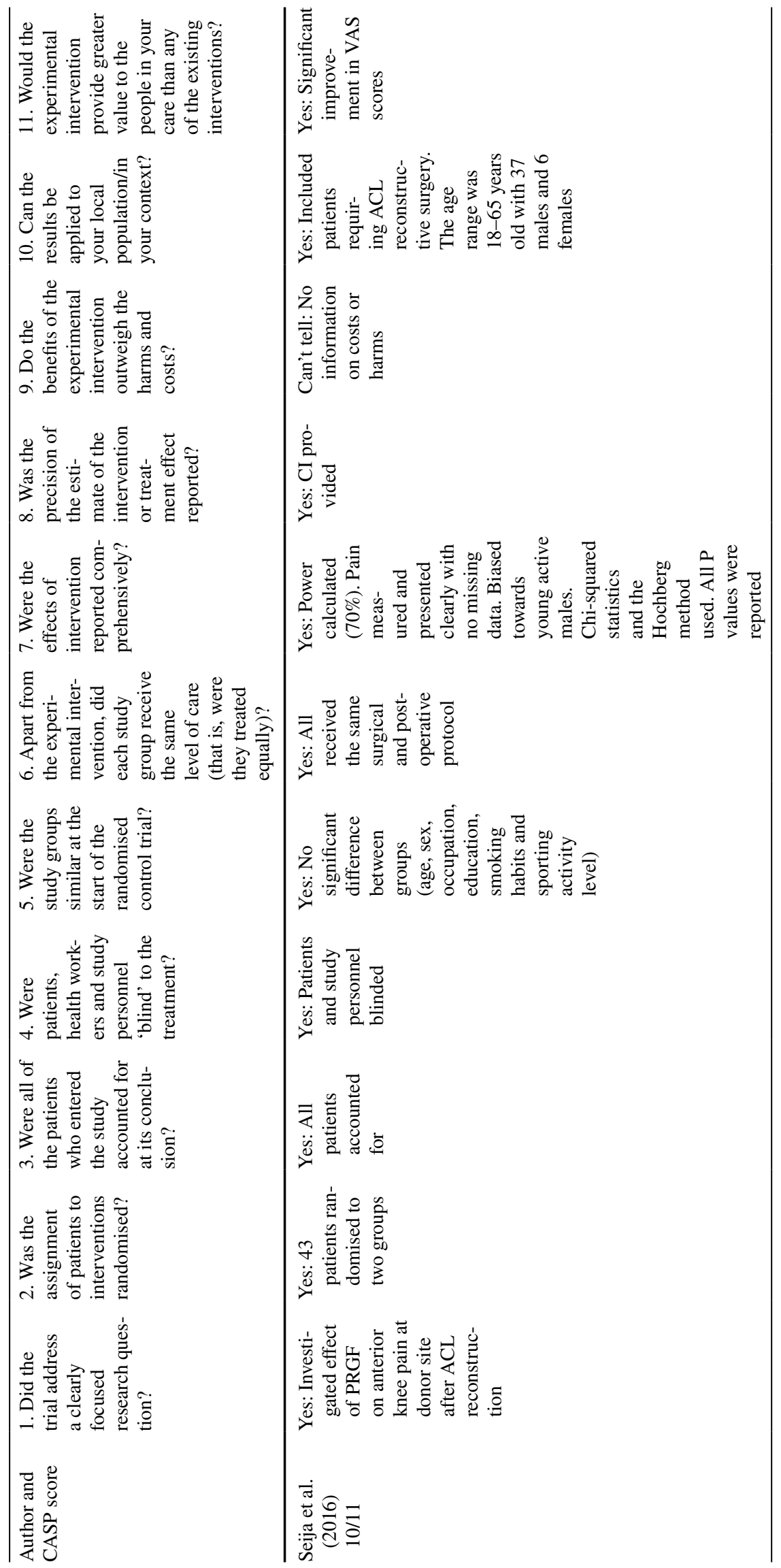




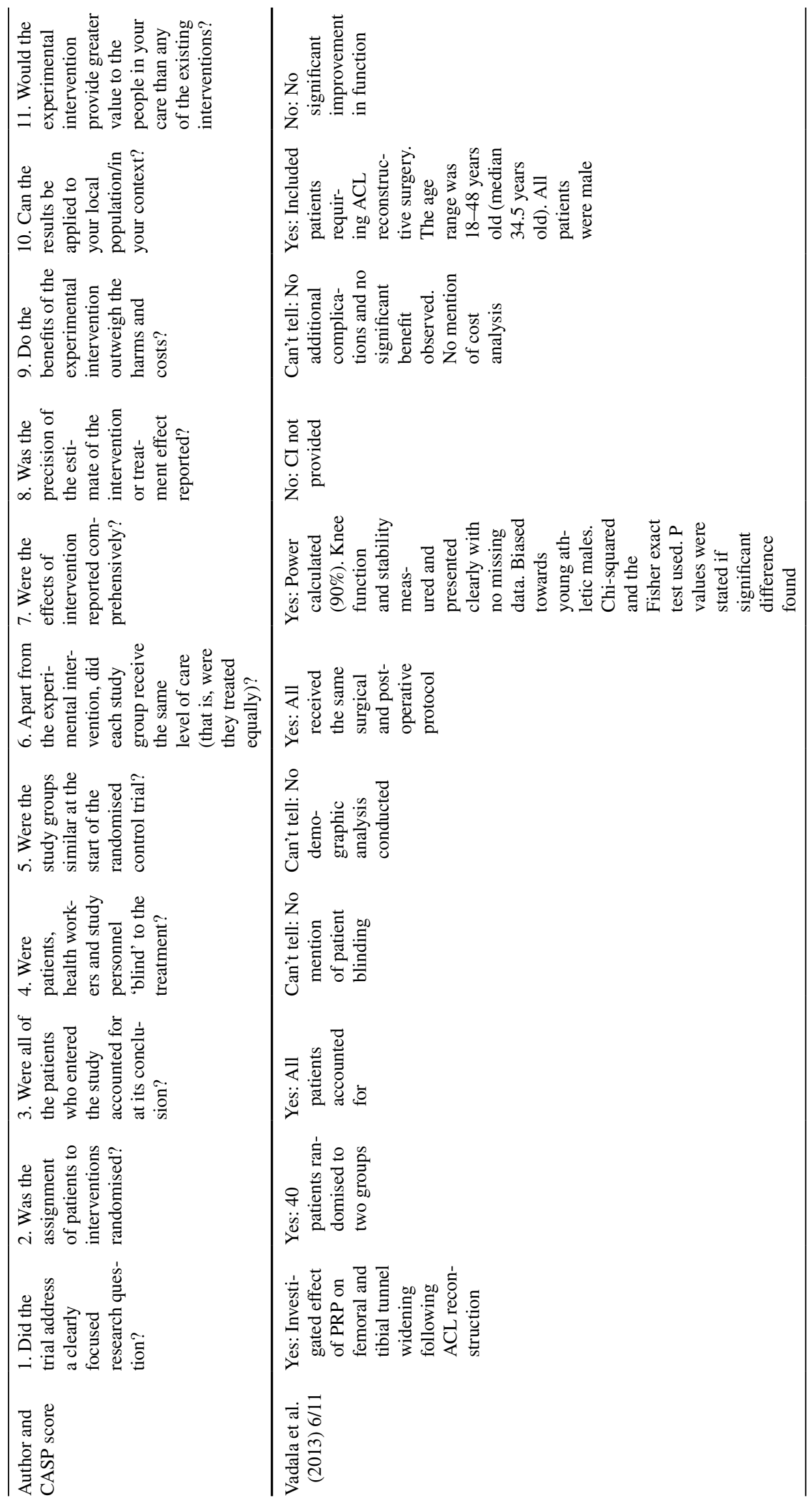




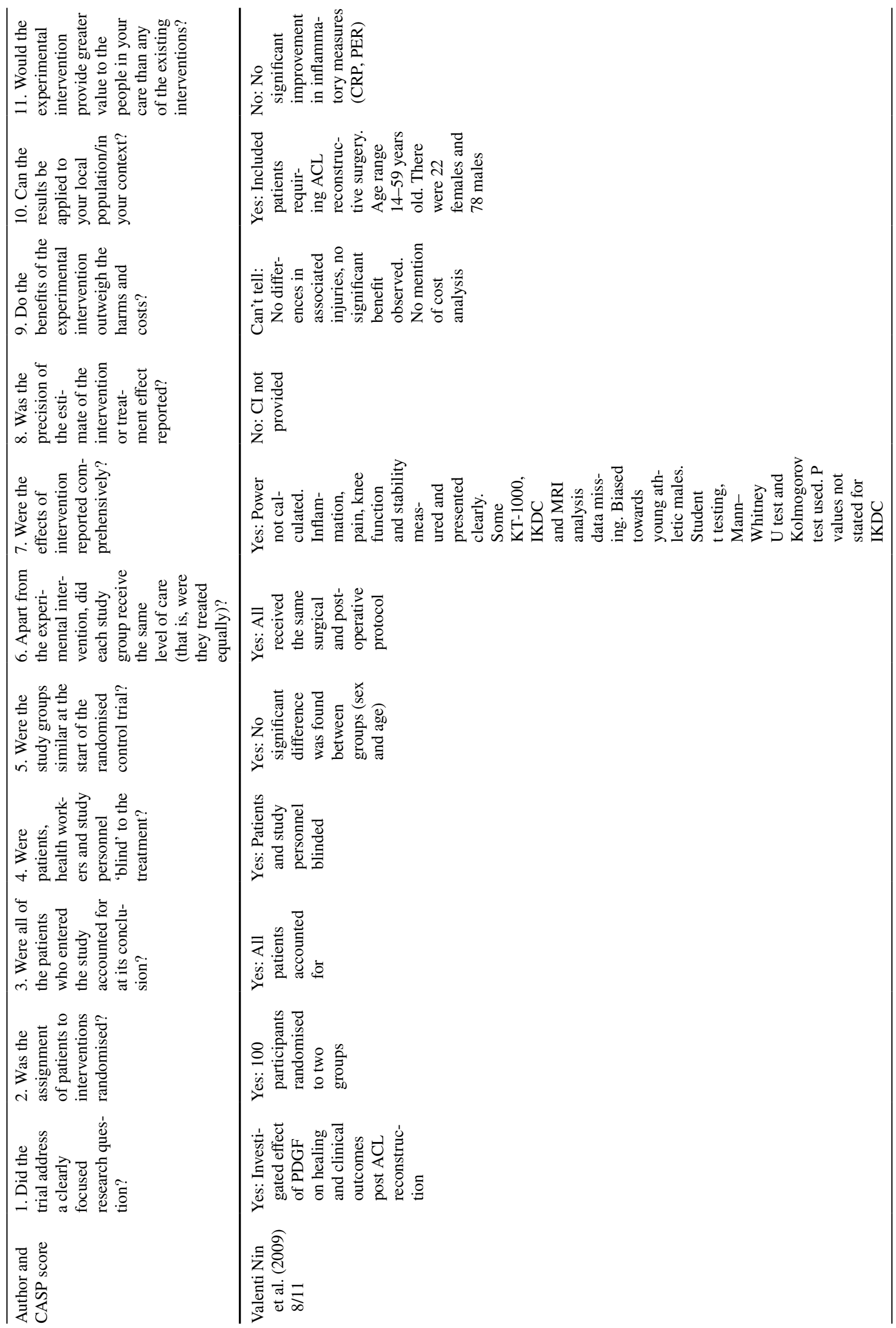




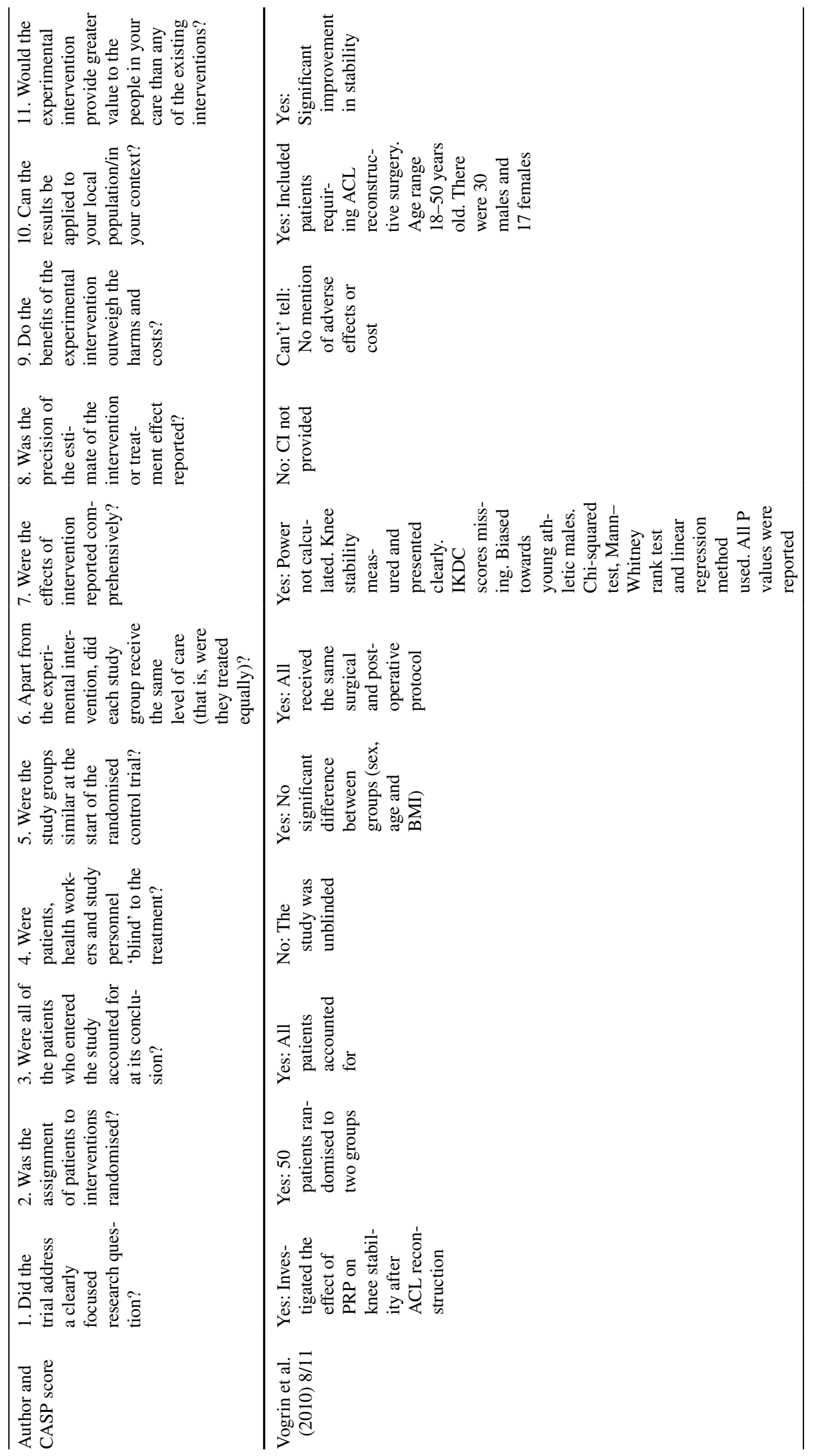




\begin{tabular}{|c|c|}
\hline 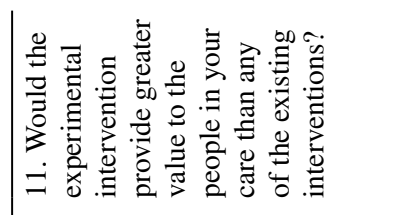 & 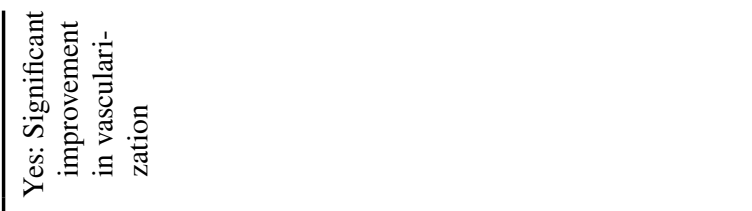 \\
\hline 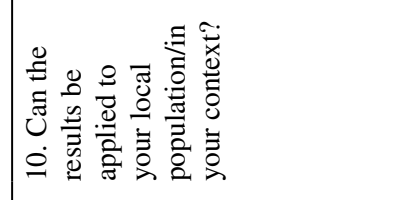 & 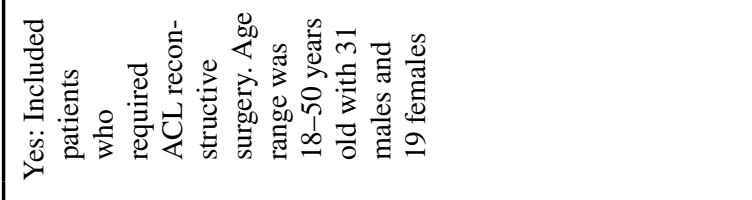 \\
\hline  & 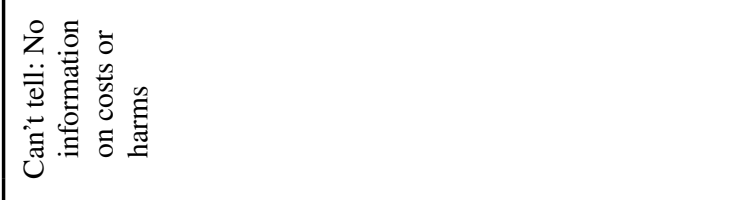 \\
\hline 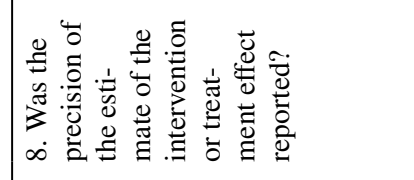 & 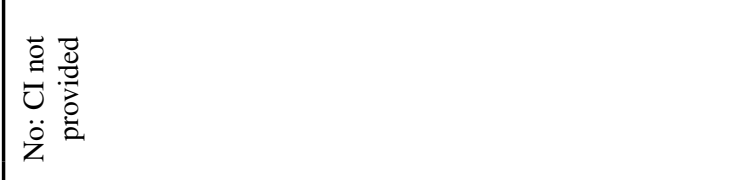 \\
\hline 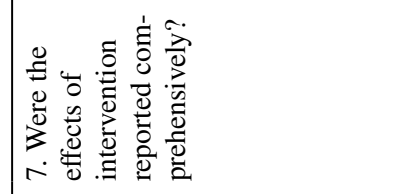 & 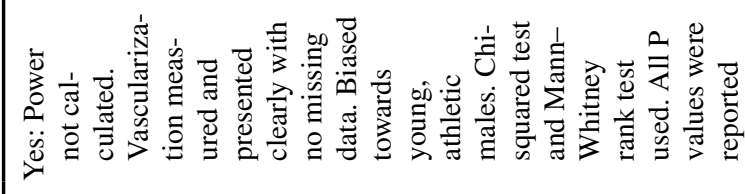 \\
\hline 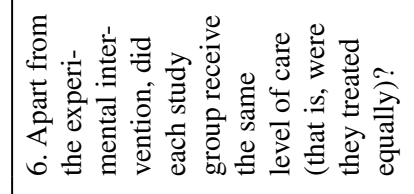 &  \\
\hline 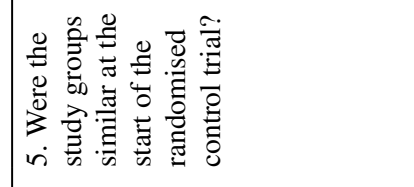 & 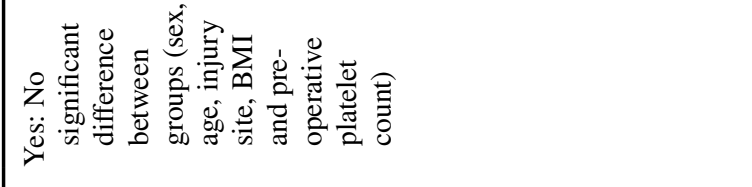 \\
\hline 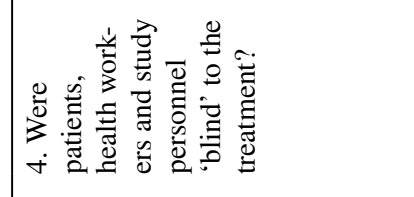 & 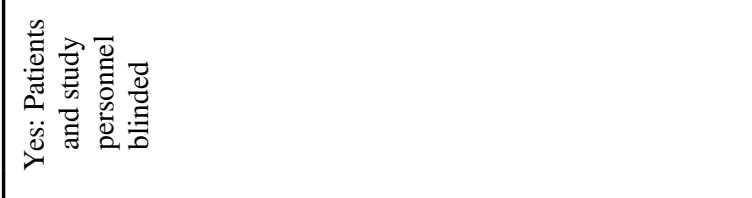 \\
\hline 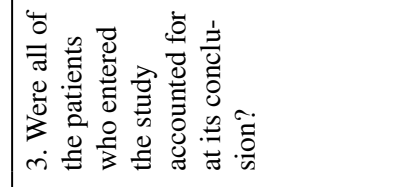 & 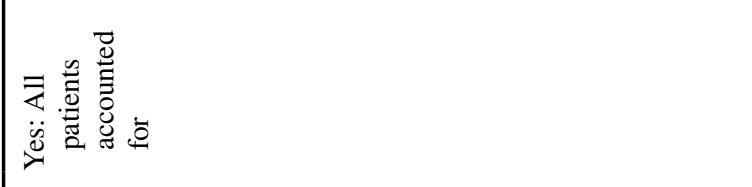 \\
\hline 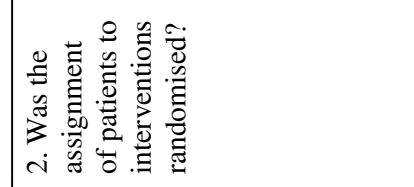 & 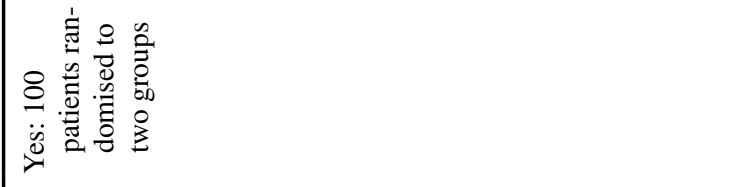 \\
\hline 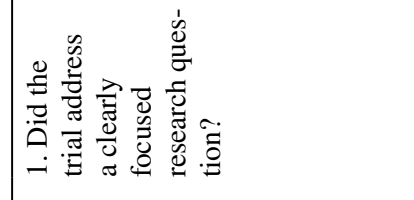 & 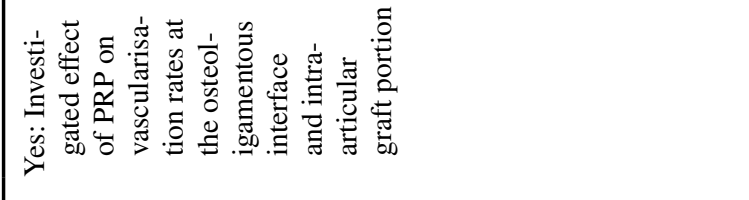 \\
\hline 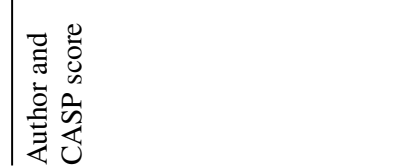 & 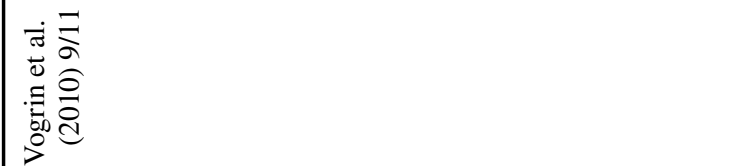 \\
\hline
\end{tabular}




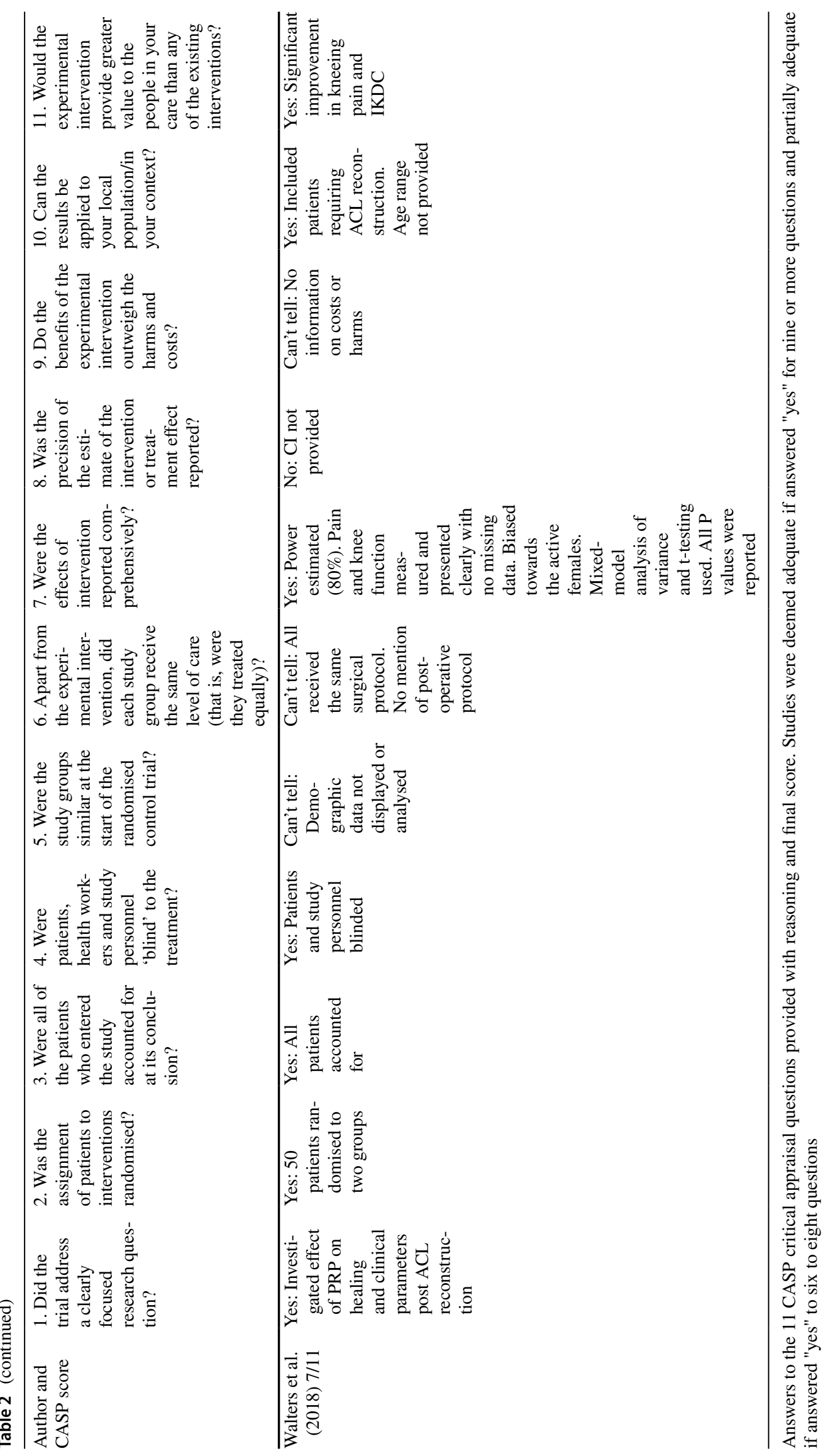




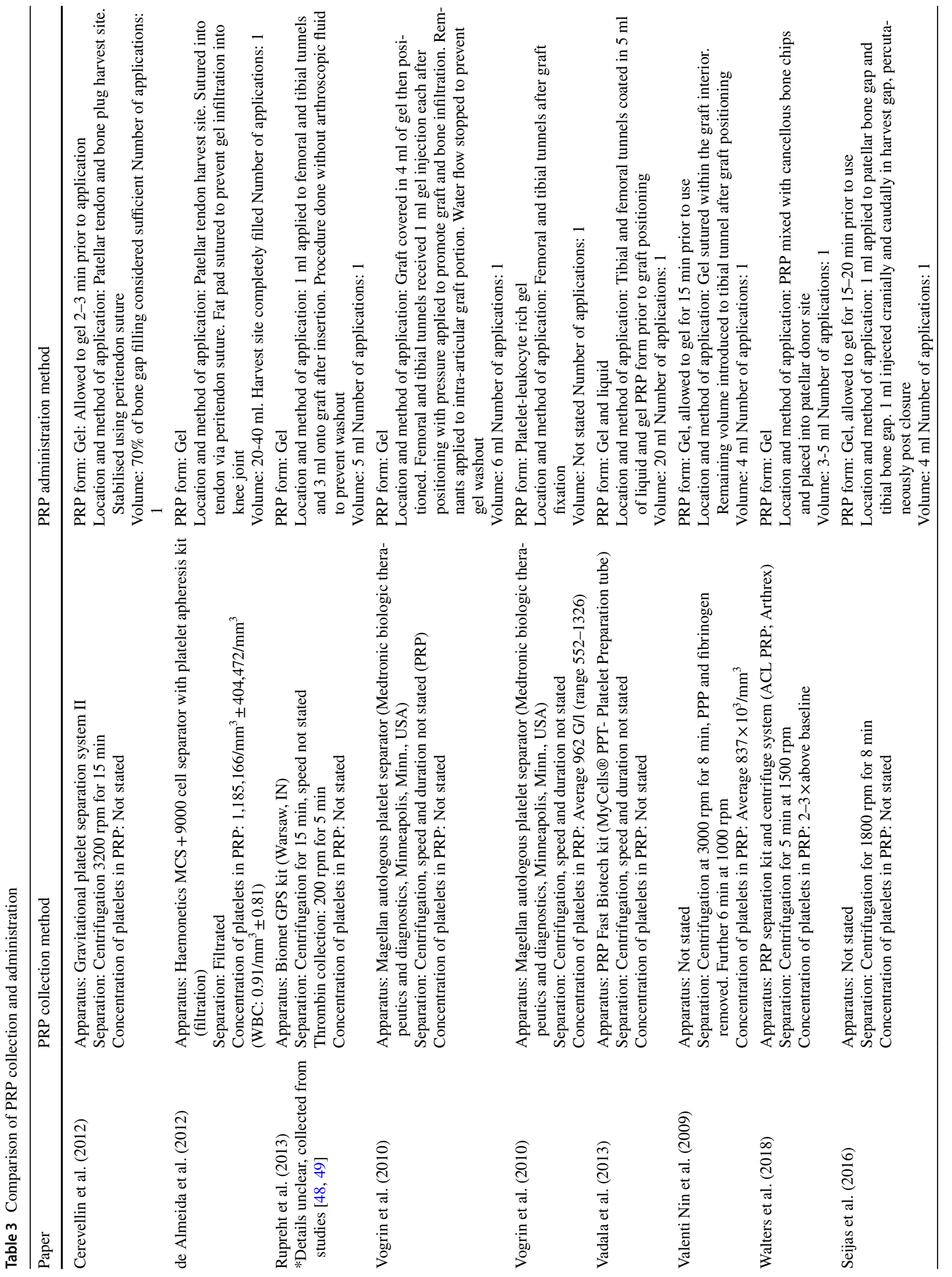


Data availability The datasets used and/or analysed during the current study are available from the corresponding author on reasonable request.

\section{Declarations}

Conflicts of interest The authors have no relevant financial or nonfinancial interests to disclose. The authors have no conflicts of interest to declare that are relevant to the content of this article. The authors have no financial or propriety interests in any material discussed in this article. All authors certify that they have no affiliations with or involvement in any organisation or entity with any financial interest of non-financial interest in the subject matter or materials discussed in this manuscript.

Ethics approval As no patients were directly involved no ethics required.

Consent to participate Not applicable.

Consent for publication Not applicable.

Open Access This article is licensed under a Creative Commons Attribution 4.0 International License, which permits use, sharing, adaptation, distribution and reproduction in any medium or format, as long as you give appropriate credit to the original author(s) and the source, provide a link to the Creative Commons licence, and indicate if changes were made. The images or other third party material in this article are included in the article's Creative Commons licence, unless indicated otherwise in a credit line to the material. If material is not included in the article's Creative Commons licence and your intended use is not permitted by statutory regulation or exceeds the permitted use, you will need to obtain permission directly from the copyright holder. To view a copy of this licence, visit http://creativecommons.org/licenses/by/4.0/.

\section{References}

1. Sanders T, Maradit Kremers H, Bryan A, Larson D, Dahm D, Levy B, Stuart M, Krych A (2016) Incidence of Anterior Cruciate Ligament Tears and Reconstruction. Am J Sports Med 44(6):1502-1507

2. Nessler T, Denney L, Sampley J (2017) ACL Injury Prevention: What Does Research Tell Us? Curr Rev Musculoskelet Med 10(3):281-288

3. Thomas A, Villwock M, Wojtys E, Palmieri-Smith R (2013) Lower Extremity Muscle Strength After Anterior Cruciate Ligament Injury and Reconstruction. J Athl Train 48(5):610-620

4. Halpern B, Chaudhury S, Rodeo S (2012) The Role of PlateletRich Plasma in Inducing Musculoskeletal Tissue Healing. HSS J 8(2):137-145

5. Martínez C, Smith P, Palma Alvarado V (2015) The influence of platelet-derived products on angiogenesis and tissue repair: a concise update. Front Physiol. https://doi.org/10.3389/fphys.2015. 00290

6. Jain N, Gulati M (2016) Platelet-rich plasma: a healing virtuoso. Blood Res 51(1):3

7. Johal H, Khan M, Yung S, Dhillon M, Fu F, Bedi A, Bhandari M (2019) Impact of Platelet-Rich Plasma Use on Pain in Orthopaedic Surgery: A Systematic Review and Meta-analysis. Sports Health: A Multidisciplinary Approach 11(4):355-366

8. Brewer B, Cornelius A, Sklar J, Van Raalte J, Tennen H, Armeli S, Corsetti J, Brickner J (2006) Pain and negative mood during 
rehabilitation after anterior cruciate ligament reconstruction: a daily process analysis. Scandinavian J Med Sci Sports. https:// doi.org/10.1111/j.1600-0838.2006.00601.x

9. la Fleur P, Argáez C., (2017) Platelet-Rich Plasma Injections for Wound Healing and Tissue Rejuvenation: A Review of Clinical Effectiveness, Cost-Effectiveness and Guidelines [Internet]. Ottawa (ON): Canadian Agency for Drugs and Technologies in Health.

10. programme CAs. CASP Randomised Controlled Trial Standard checklist [Available from: https://casp-uk.b-cdn.net/wp-content/ uploads/2020/10/CASP_RCT_Checklist_PDF.pdf]

11. Cervellin M, de Girolamo L, Bait C, Denti M, Volpi P (2011) Autologous platelet-rich plasma gel to reduce donor-site morbidity after patellar tendon graft harvesting for anterior cruciate ligament reconstruction: A randomized, controlled clinical study. Knee Surg Sports Traumatol Arthrosc 20(1):114-120

12. Lopez-Vidriero E, Olive-Vilas R, Lopez-Capape D, Varela-Sende L, Lopez-Vidriero R, Til-Perez L (2019) Efficacy and Tolerability of Progen, a Nutritional Supplement Based on Innovative Plasma Proteins, in ACL Reconstruction: A Multicenter Randomized Controlled Trial. Orthop J Sports Med 7(2):232596711982723

13. Mirzatolooei F, Alamdari M, Khalkhali H (2013) The impact of platelet-rich plasma on the prevention of tunnel widening in anterior cruciate ligament reconstruction using quadrupled autologous hamstring tendon: A randomised clinical trial. J Bone Joint Surg Series B 95(1):65-69

14. Vogrin M, Rupreht M, Crnjac A, Dinevski D, Krajnc Z, Recnik G (2010) The effect of platelet-derived growth factors on knee stability after anterior cruciate ligament reconstruction: A prospective randomized clinical study. Wien Klin Wochenschr 122(SUPPL. 2):91-95

15. de Almeida AM, Demange M, Sobrado M, Rodrigues M, Pedrinelli A, Hernandez A (2012) Patellar tendon healing with platelet-rich plasma: a prospective randomized controlled trial. Am J Sports Med 40(6):1282-1288

16. Mahdi M, Halwas Jhale H (2019) Value of Platelet-Rich Plasma in Osteointegration of Tendon Graft in Anterior Cruciate Ligament Reconstruction. Res J Med Sci 13(4):76-81

17. Vadala A, Iorio R, De Carli A, Ferretti M, Paravani D, Caperna L, Iorio C, Gatti A, Ferretti A (2012) Platelet-rich plasma: Does it help reduce tunnel widening after ACL reconstruction? Knee Surg Sports Traumatol Arthrosc 21(4):824-829

18. Walters BL, Porter D, Hobart S, Bedford B, Hogan D, McHugh M, Klein D, Harousseau K, Nicholas S (2018) Effect of Intraoperative Platelet-Rich Plasma Treatment on Postoperative Donor Site Knee Pain in Patellar Tendon Autograft Anterior Cruciate Ligament Reconstruction: A Double-Blind Randomized Controlled Trial. Am J Sports Med 46(8):1827-1835

19. Rupreht M, Vogrin M, Hussein M (2013) Evaluation of the tibial tunnel after intraoperatively administered platelet-rich plasma gel during anterior cruciate ligament reconstruction using diffusion weighted and dynamic contrast-enhanced MRI. J Magn Reson Imaging 37(4):928-935

20. Seijas R, Cusco X, Sallent A, Serra I, Ares O, Cugat R (2016) Pain in donor site after BTB-ACL reconstruction with PRGF: a randomized trial. Arch Orthop Trauma Surg 136(6):829-835

21. Vogrin M, Rupreht M, Dinevski D, Haspl M, Kuhta M, Jevsek M, Knezevic M, Rozman P (2010) Effects of a Platelet Gel on Early Graft Revascularization after Anterior Cruciate Ligament Reconstruction: A Prospective, Randomized Double-Blind. Clin Trial Eur Surg Res 45(2):77-85

22. Valentí Nin J, Mora Gasque G, Valentí Azcárate A, Aquerreta Beola J, Hernandez Gonzalez M (2009) Has PlateletRich Plasma Any Role in Anterior Cruciate Ligament Allograft Healing? Arthroscopy J Arthroscopic Related Surg 25(11):1206-1213
23. Haefeli M, Elfering A (2005) Pain assessment. Eur Spine J 15(S1):S17-S24

24. Katz J, Fingeroth R (1986) The diagnostic accuracy of ruptures of the anterior cruciate ligament comparing the Lachman test, the anterior drawer sign, and the pivot shift test in acute and chronic knee injuries. Am J Sports Med 14(1):88-91

25. Collins N, Misra D, Felson D, Crossley K, Roos E (2011) Measures of knee function: International Knee Documentation Committee (IKDC) Subjective Knee Evaluation Form, Knee Injury and Osteoarthritis Outcome Score (KOOS), Knee Injury and Osteoarthritis Outcome Score Physical Function Short Form (KOOS-PS). Knee Ou Arthritis Care Res 63(S11):S208-S228

26. Tegner Y, Lysholm J (1985) Rating Systems in the Evaluation of Knee Ligament Injuries. Clin Orthop Relat Res 198:43-49

27. Hernandez-Sanchez S, Abat F, Hidalgo M, Cuesta-Vargas A, Segarra V, Sanchez-Ibañez J, Gomez-Conesa A (2017) Confirmatory factor analysis of VISA-P scale and measurement invariance across sexes in athletes with patellar tendinopathy. J Sport Health Sci 6(3):365-371

28. Seijas R, Ares O, Catala J, Alvarez-Diaz P, Cusco X, Cugat R (2013) Magnetic resonance imaging evaluation of patellar tendon graft remodelling after anterior cruciate ligament reconstruction with or without platelet-rich plasma. J Orthop Surg (Hong Kong) 21(1):10-14

29. Kidd B, Urban L (2001) Mechanisms of inflammatory pain. Br J Anaesth 87(1):3-11

30. Han F, Banerjee A, Shen L, Krishna L (2015) Increased Compliance With Supervised Rehabilitation Improves Functional Outcome and Return to Sport After Anterior Cruciate Ligament Reconstruction in Recreational Athletes. Orthop J Sports Med 3(12):232596711562077

31. Sánchez M, Delgado D, Sánchez P, Fiz N, Azofra J, Orive G, Anitua E, Padilla S (2014) Platelet Rich Plasma and Knee Surgery. Biomed Res Int 2014:1-10

32. Mendias C, Lynch E, Davis M, Sibilsky Enselman E, Harning J, DeWolf P, Makki T, Bedi A (2013) Changes in Circulating Biomarkers of Muscle Atrophy, Inflammation, and Cartilage Turnover in Patients Undergoing Anterior Cruciate Ligament Reconstruction and Rehabilitation. Am J Sports Med 41(8):1819-1826

33. Anitua E, Andia I, Ardanza B, Nurden P, Nurden AT (2004) Autologous platelets as a source of proteins for healing and tissue regeneration. Thromb Haemost 91(1):4-15

34. Deehan D, Cawston T (2005) The biology of integration of the anterior cruciate ligament. The Journal of Bone and Joint Surgery. British 87:889-895

35. Janssen R, Scheffler S (2013) Intra-articular remodelling of hamstring tendon grafts after anterior cruciate ligament reconstruction. Knee Surg Sports Traumatol Arthrosc 22(9):2102-2108

36. Azcarate A, Lamo-Espinosa J, Beola J, Gonzalez M, Gasque G, Nin J (2014) Comparison between two different platelet-rich plasma preparations and control applied during anterior cruciate ligament reconstruction. Is there any evidence to support their use? Injury 45:S36-S41

37. Theoret, C. and Stashak, T., (2014). Integumentary System. Equine Emergencies, pp.238-267.

38. Claes S, Verdonk P, Forsyth R, Bellemans J (2011) The "Ligamentization" Process in Anterior Cruciate Ligament Reconstruction. Am J Sports Med 39(11):2476-2483

39. Matsui M, Tabata Y (2012) Enhanced angiogenesis by multiple release of platelet-rich plasma contents and basic fibroblast growth factor from gelatin hydrogels. Acta Biomater 8(5):1792-1801

40. Silva A, Sampaio R (2009) Anatomic ACL reconstruction: Does the platelet-rich plasma accelerate tendon healing? Knee Surg Sports Traumatol Arthrosc 17(6):676-682 
41. Unterhauser F, Bail H, Hoher J, Haas N, Weiler A (2003) Endoligamentous Revascularization of an Anterior Cruciate Ligament Graft. Clin Orthop Relat Res 414:276-288

42. Webster K, Feller J (2019) A research update on the state of play for return to sport after anterior cruciate ligament reconstruction. J Orthopaedics Traumatol. https://doi.org/10.1186/ s10195-018-0516-9

43. Myer G, Ford K, Paterno M, Nick T, Hewett T (2008) The Effects of Generalized Joint Laxity on Risk of Anterior Cruciate Ligament Injury in Young Female Athletes. Am J Sports Med 36(6): 1073-1080

44. Tavassoli M, Janmohammadi N, Hosseini A, Khafri S, Esmaeilnejad-Ganji S (2019) Single- and double-dose of platelet-rich plasma versus hyaluronic acid for treatment of knee osteoarthritis: A randomized controlled trial. World J Orthopedics 10(9):310-326

45. Filbay S, Grindem H (2019) Evidence-based recommendations for the management of anterior cruciate ligament (ACL) rupture. Best Pract Res Clin Rheumatol 33(1):33-47

46. Moses B, Orchard J, Orchard J (2012) Systematic Review: Annual Incidence of ACL Injury and Surgery in Various Populations. Res Sports Med 20(3-4):157-179
47. Montalvo A, Schneider D, Yut L, Webster K, Beynnon B, Kocher M, Myer G (2018) "What's my risk of sustaining an ACL injury while playing sports?" A systematic review with meta-analysis. Br J Sports Med 53(16):1003-1012

48. Orrego M, Larrain C, Rosales J, Valenzuela L, Matas J, Durruty J, Sudy H, Mardones R (2008) Effects of Platelet Concentrate and a Bone Plug on the Healing of Hamstring Tendons in a Bone Tunnel. Arthroscopy J Arthroscopic Related Surg 24(12):1373-1380

49. Radice F, Yánez R, Gutiérrez V, Rosales J, Pinedo M, Coda S (2010) Comparison of Magnetic Resonance Imaging Findings in Anterior Cruciate Ligament Grafts With and Without Autologous Platelet-Derived Growth Factors. Arthroscopy J Arthroscopic Related Surg 26(1):50-57

Publisher's Note Springer Nature remains neutral with regard to jurisdictional claims in published maps and institutional affiliations. 